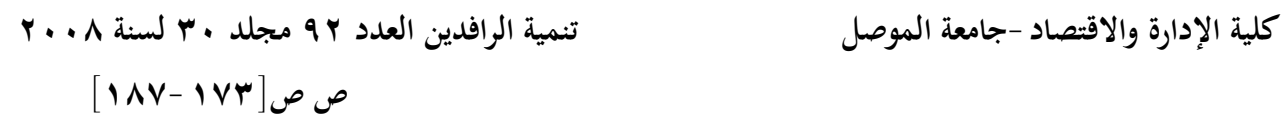

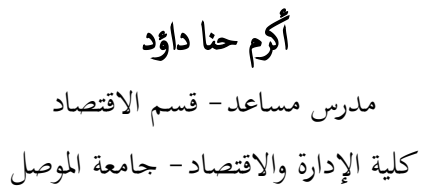

Akrm_aboalin@yahoo.com

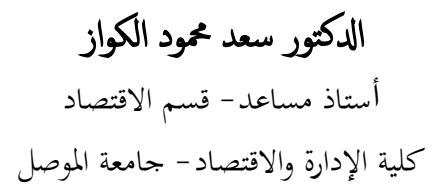

Saad_alkawaz@yahoo.com

\title{
المستخلص
}

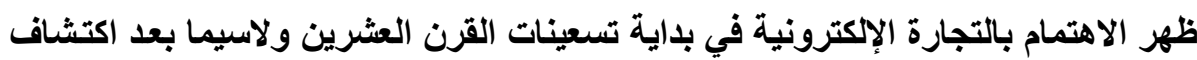

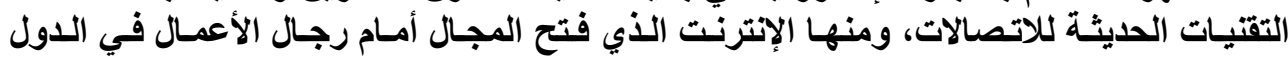

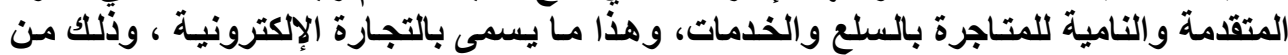

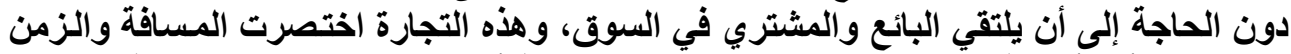

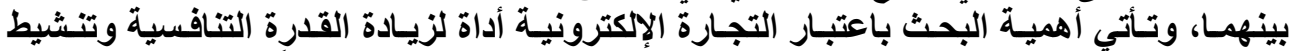

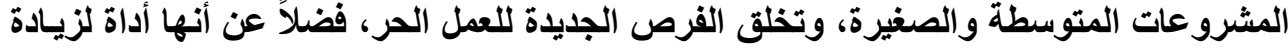

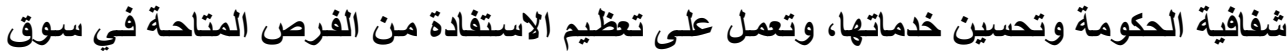

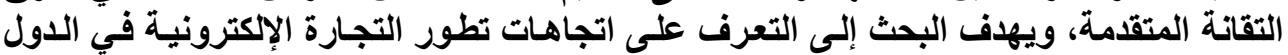

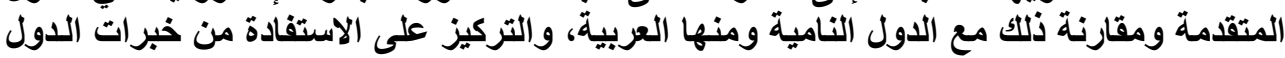

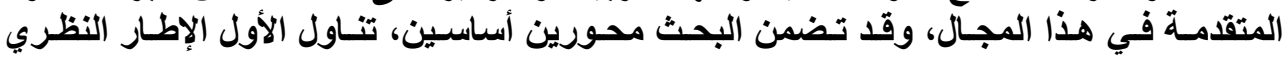

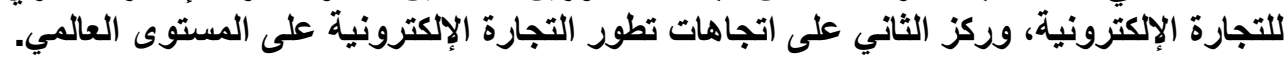

\section{The Trends of Development the Electronic Commerce with Reference to the Arab Countries}

\author{
Saad M. Al - Kawwaz (PhD) \\ Assistant Professor \\ University of Mosul
}

\author{
Akram H. Dawood \\ Assistant Lecturer \\ University of Mosul
}

Abstract

The importance of the electronic commerce has recently been realized during the 1990s of 20 century after modern technologies of communications were uncovered. Internet technology was among them that have been propounded to the businessmen in the developed and growing countries to trade out the goods and services. This can be said to be the electronic commerce. The notion needs not to meet both purchaser and buyer in the markets. This model of commerce may not take

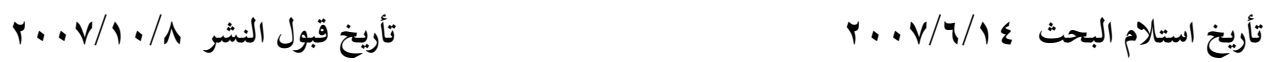


both time and distance at application. The importance of the current paper refers that electronic commerce is a way of increasing the competitive ability as to activate the small and middle projects and recreate new choices for free works, in addition to increase the strategy of the government transparently and enlarge the choices of progressed technology markets. The researches aims at explaining the trends of government development in the various developed countries and compare that with under development and Arab countries. The developed countries experiences can be centered within two aspects:

- The first one included the theoretical background to the electronic commerce.

- The second focused on the trends of electronic commerce development globally.

المقدمة

تعد التجارة الإلكترونية إحدى النتاجات المهمة للثورة التكنولوجية و المعلوماتية

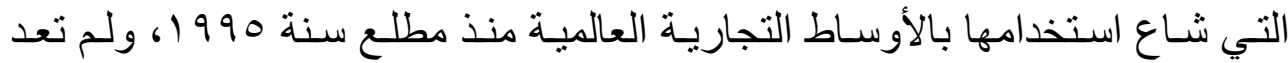

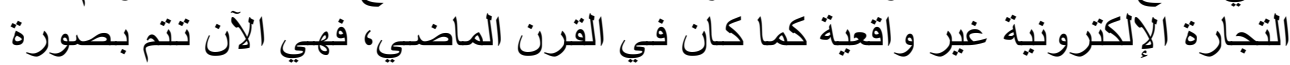

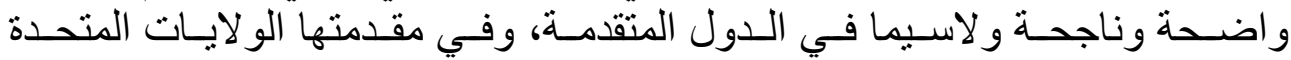

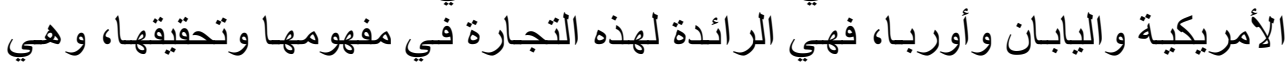

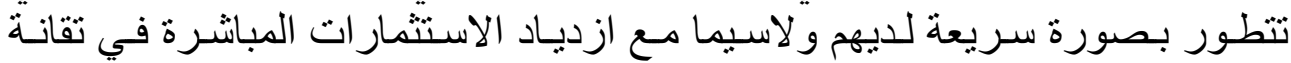

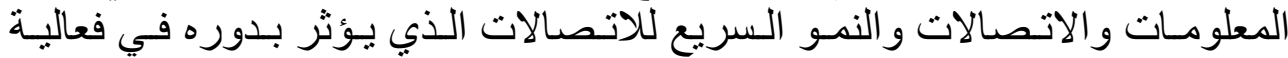

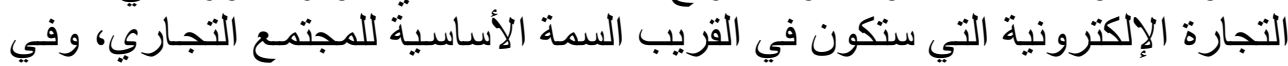

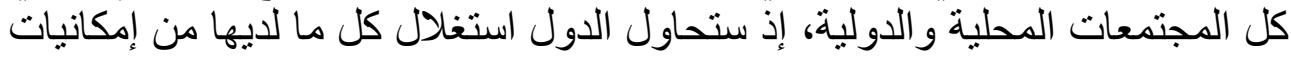

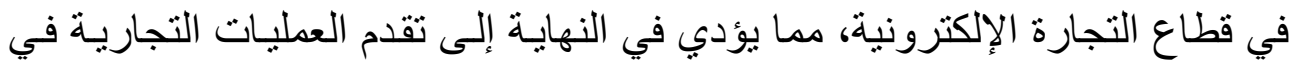
هذّه الدول.

مشكنة البحث

تتلخص مشكلة البحث بالتساؤل الآتي: هل يمكن للاول المتقدمة مساعدة الدول

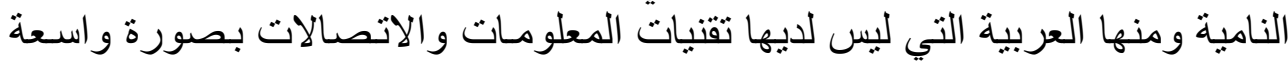

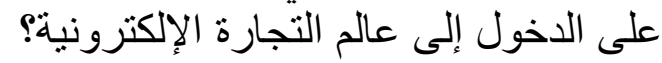
هدف البحث

يهـدف البحـث التعرف إلـى اتجاهـات تطـور التجـارة الإلكترونيـة في الـدول المتقدمة ومقارنة ذلك مع الدول النامية ومنها العربية. فرضية البحثة

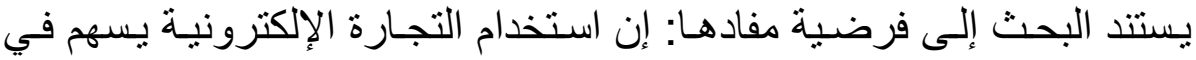

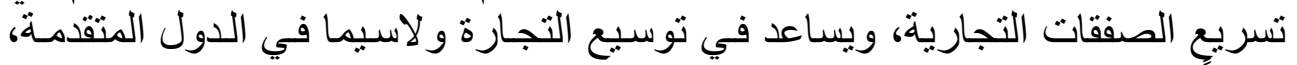

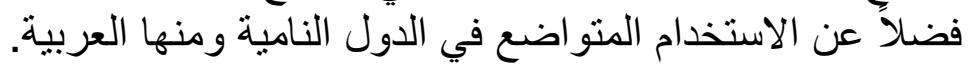

منهج البحث

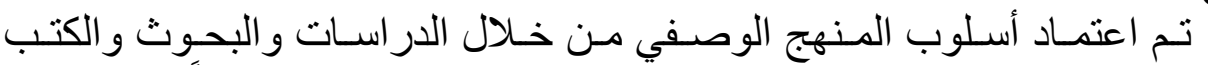

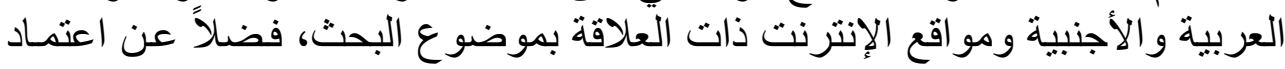

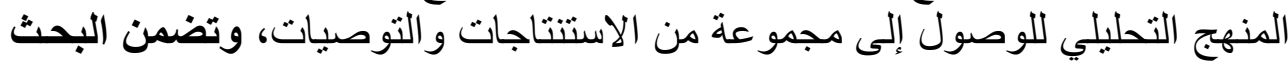


محورين أساسين تنـاول الأول الإطـار النظري للتجارة الإلكترونيـة، أمسا الثاني فقد

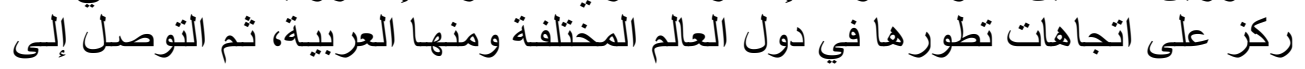

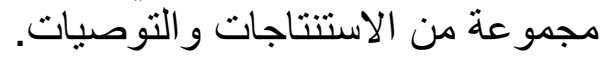

\section{المحور الأول- الإطار النظري للتجارة الإلكترونية}

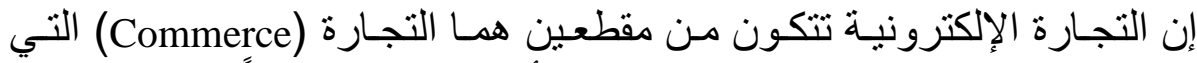

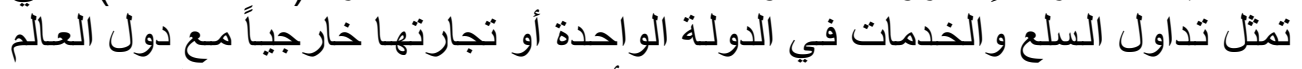

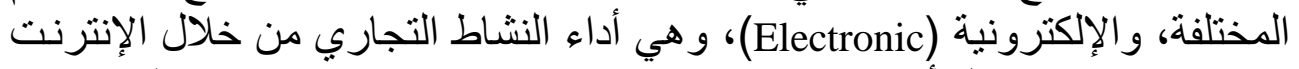

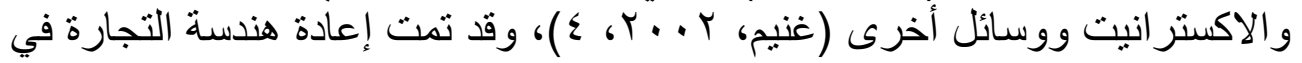

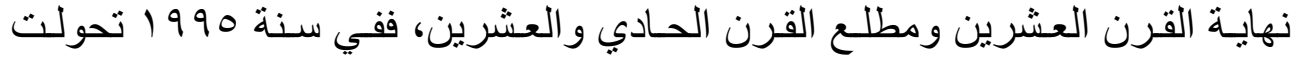

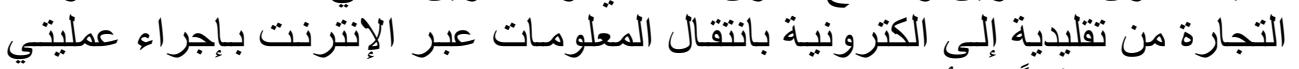

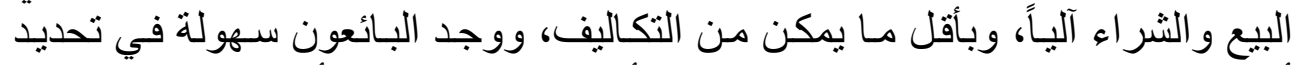

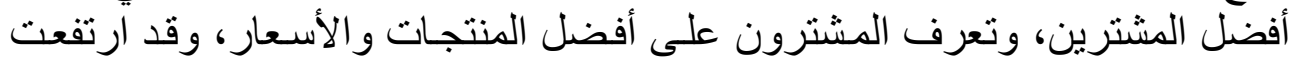

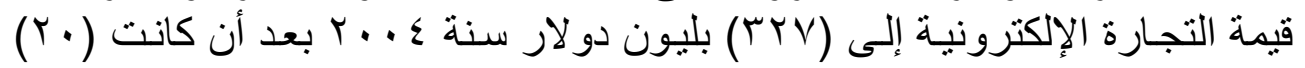

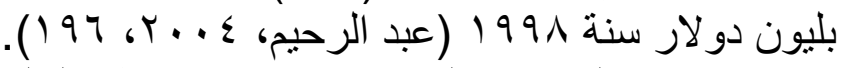

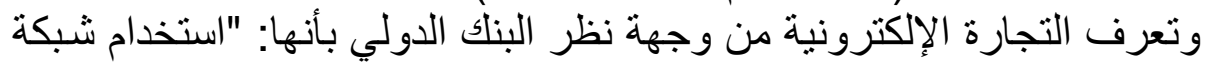

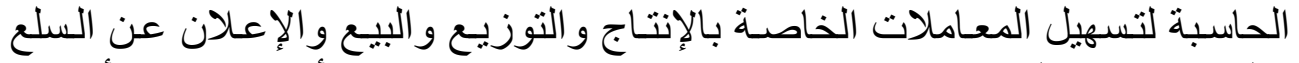

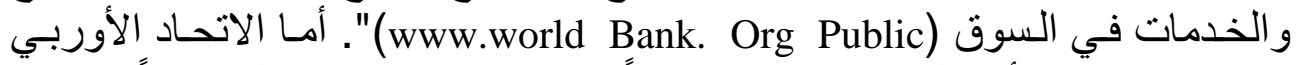
فيعتبر ها إجر أَ الأعمال التجاريـة إلكترونياً (www.Eu.org)، وتمثنل نظامـاً يستخدم

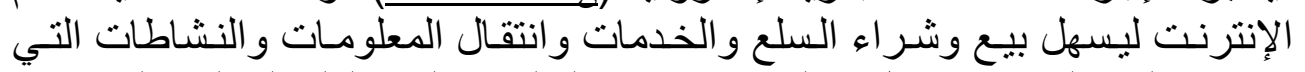

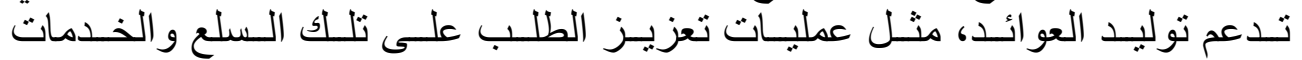

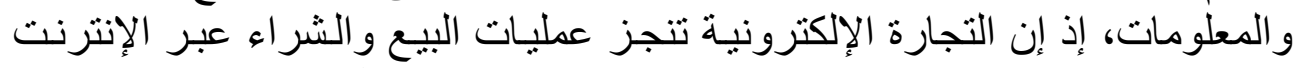

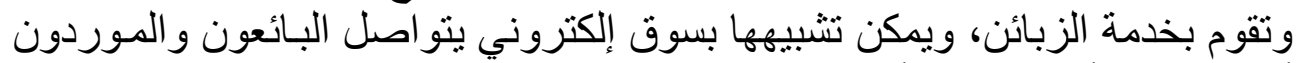

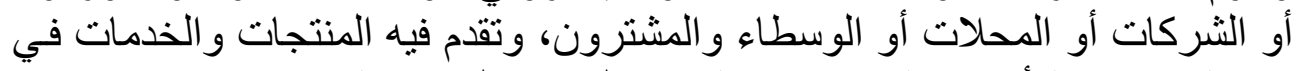
صيغة افتر اضية أو رقمية، كما يدفع ثمنها بـالنقود الإلكترونيـة (www.alnahdi:ws).

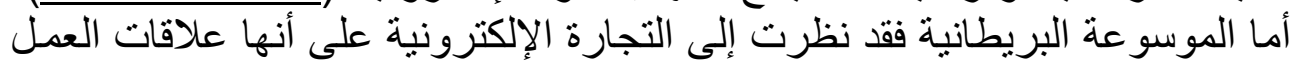

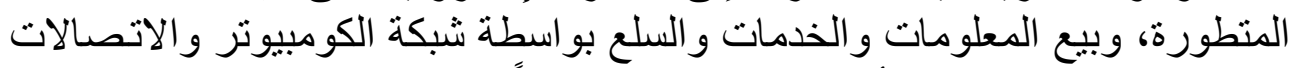
(www.britinlca.com)

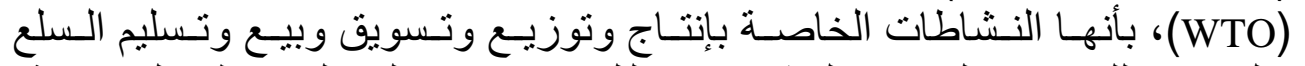
و الخدمات للمستخدم النهائي (المشتري) وذللك بالاعتمـاد على الألى الوسـائط الإلكترونية،

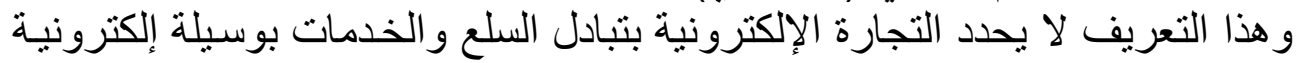

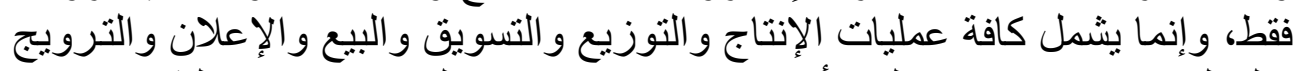

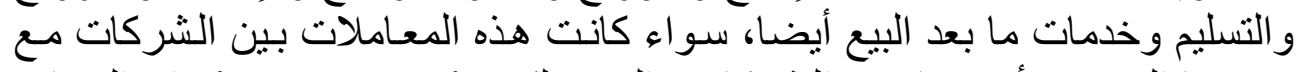

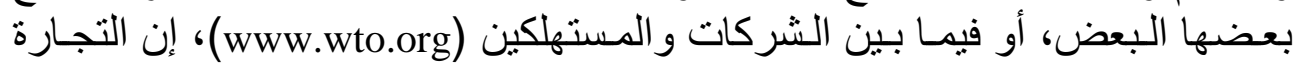

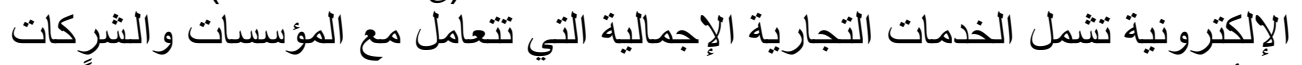

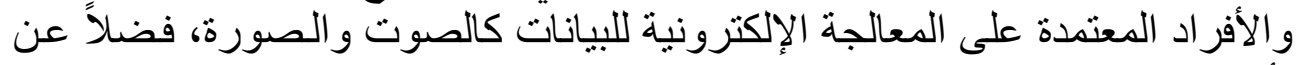

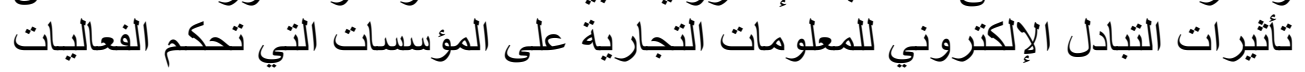




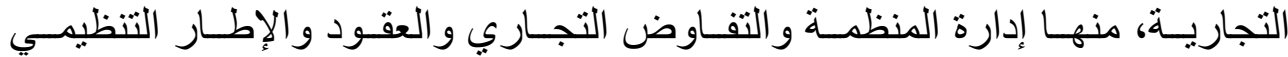

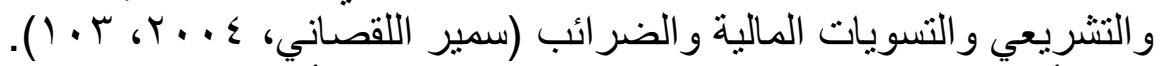

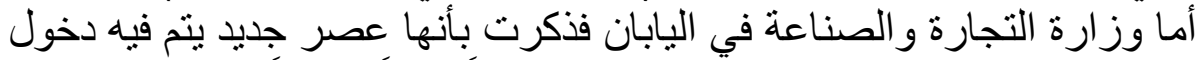

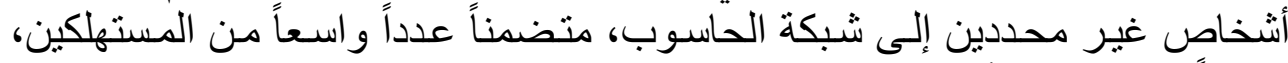

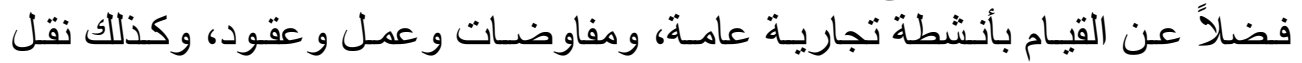

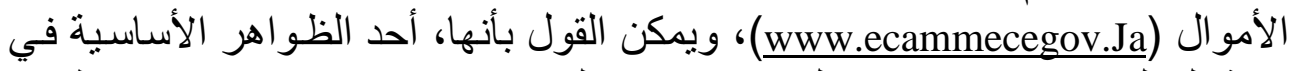

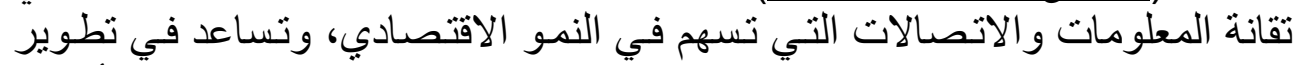

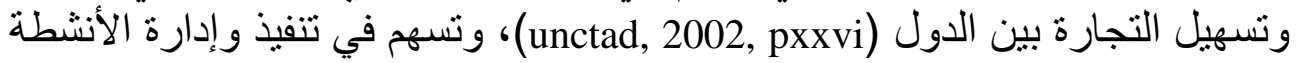

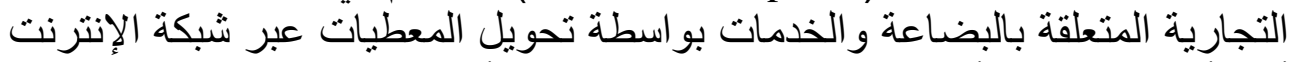

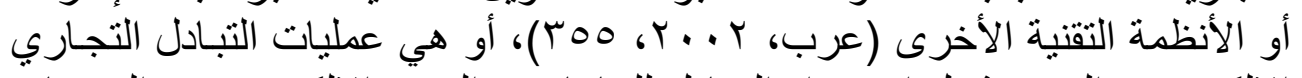

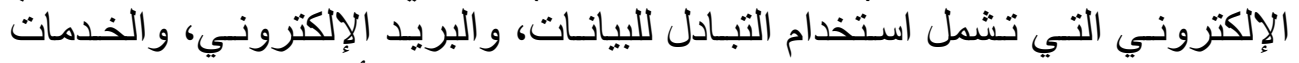

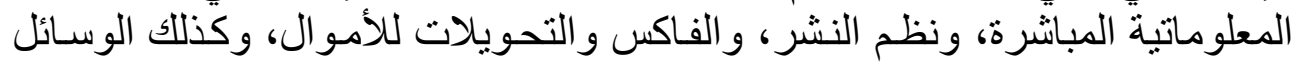
الإلكترونية الأخرى. (Shelly, et, al, 2004, 10-14)

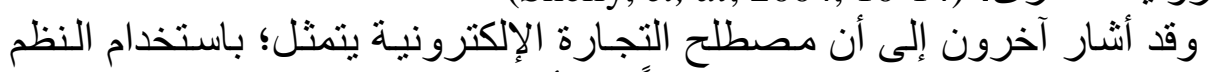

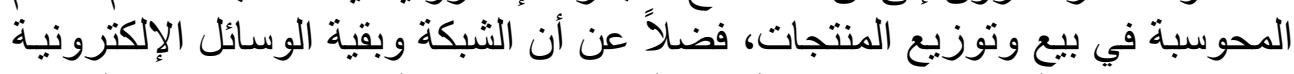

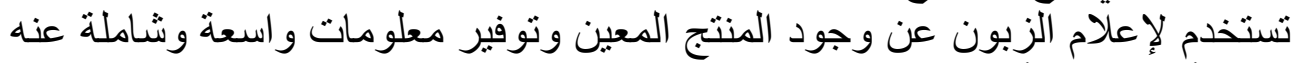

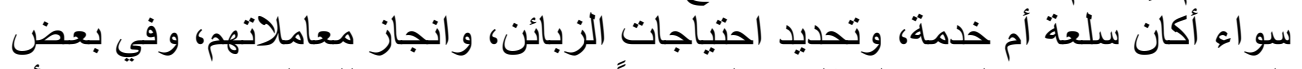

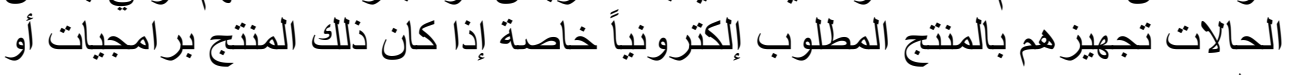
اتسمت التجارة الإلكترونية بخصائص متعددة نتيجة استخدام تقانة المعلومات التهات معلومات (Alter, 1999, 204).

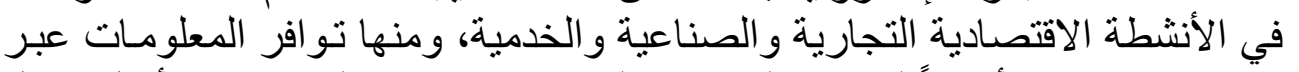

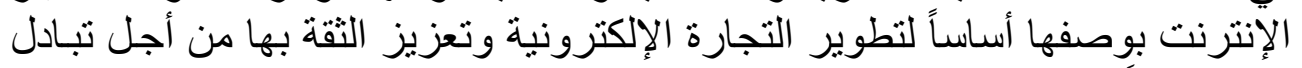

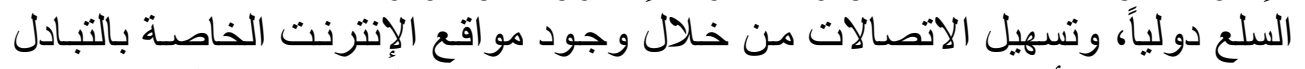

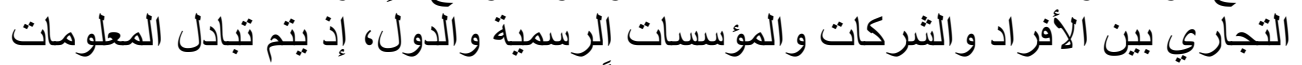

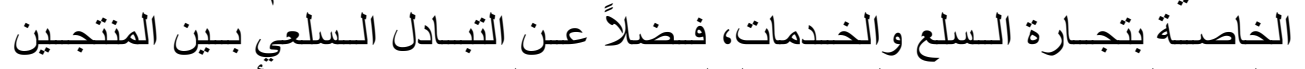

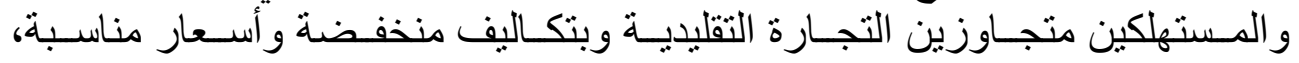

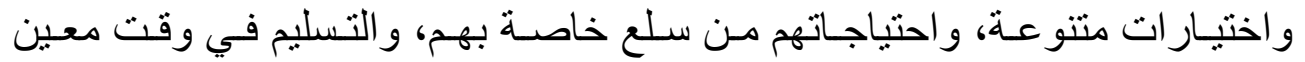

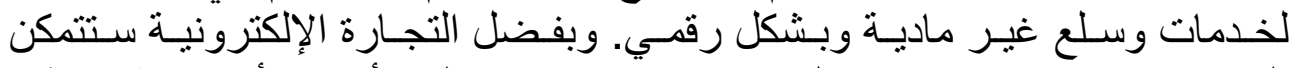

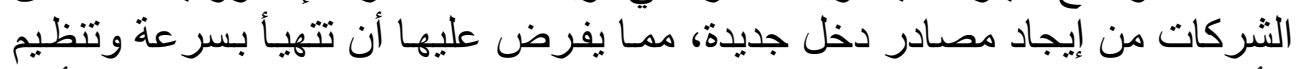

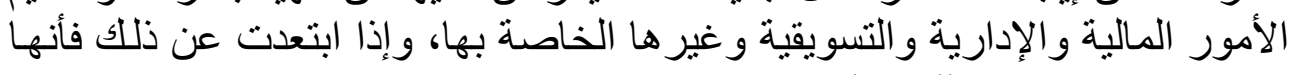

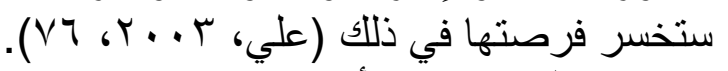

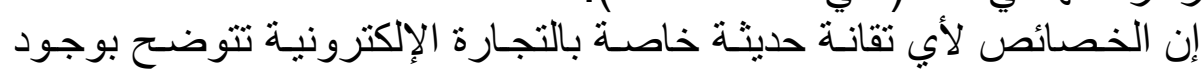

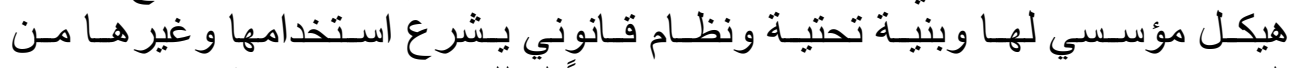

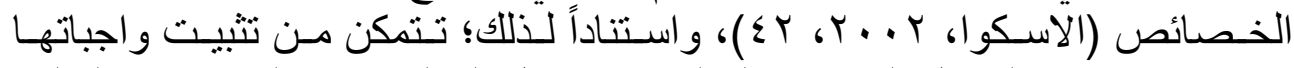

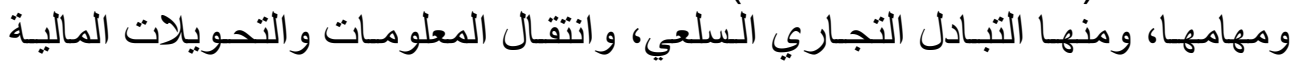

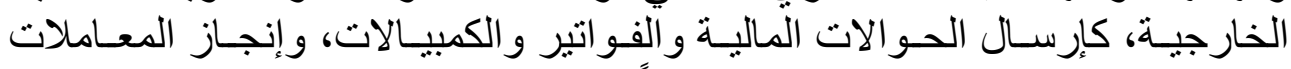

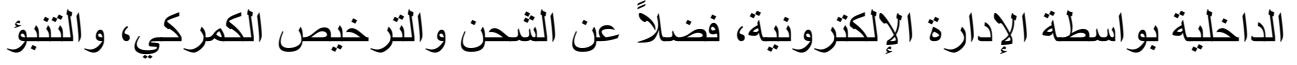




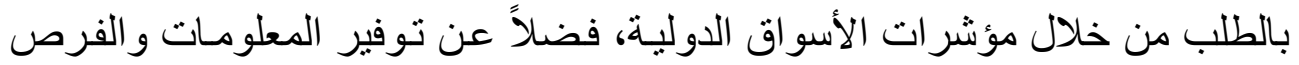

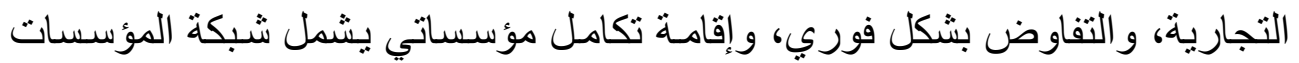

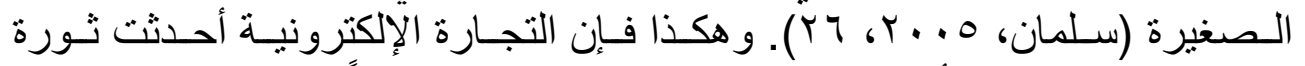

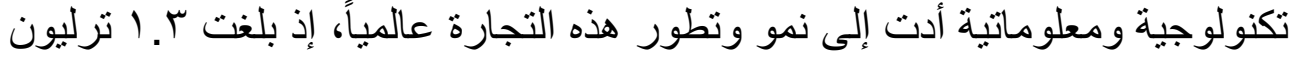

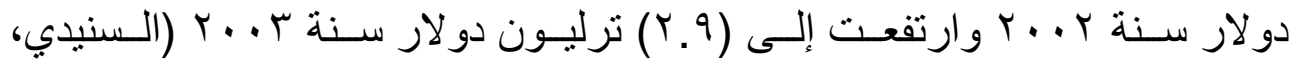

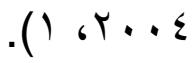

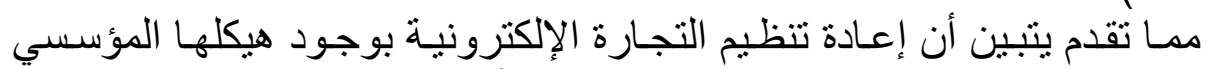

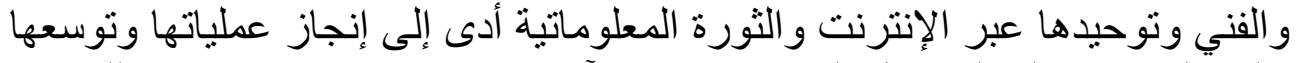

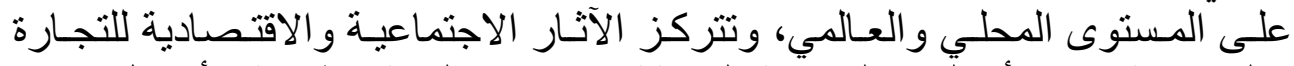

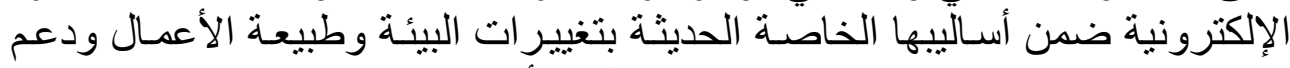

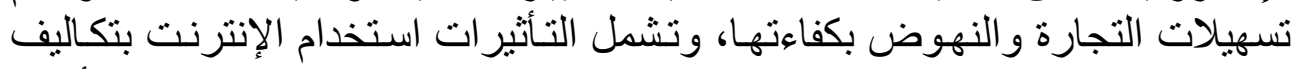

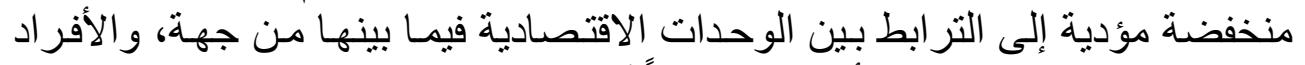

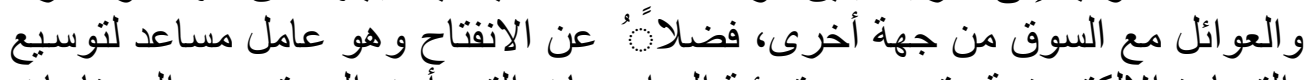

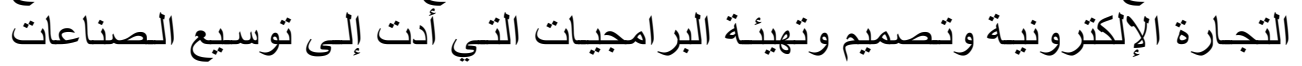

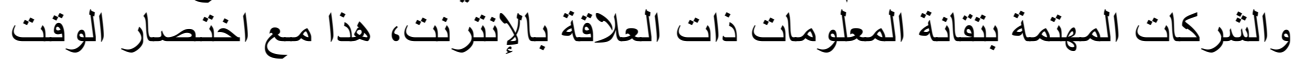

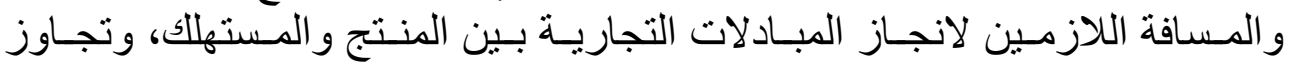

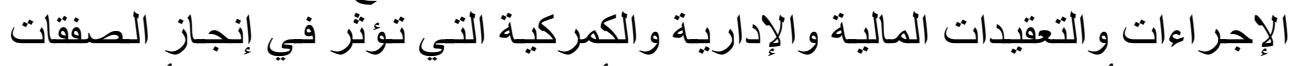

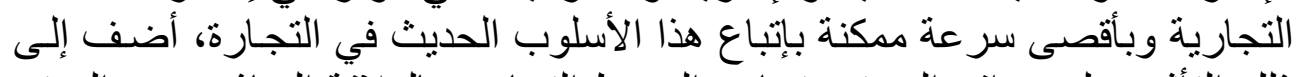

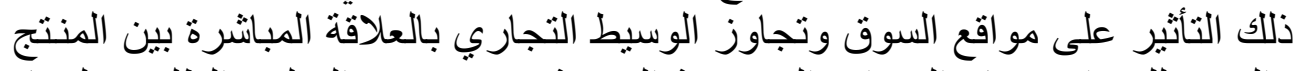

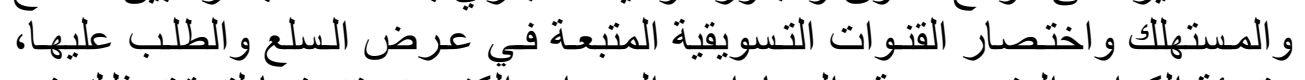

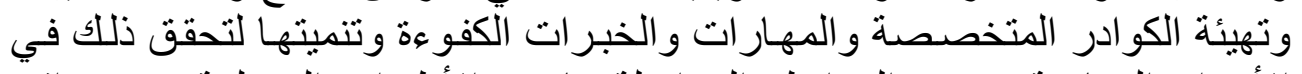

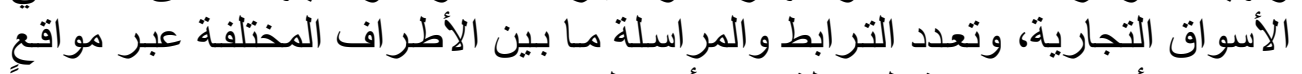

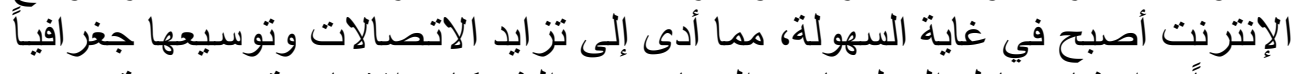

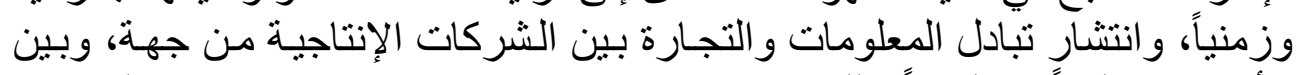

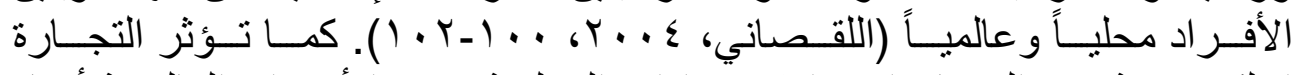

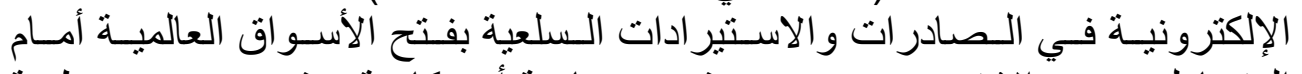

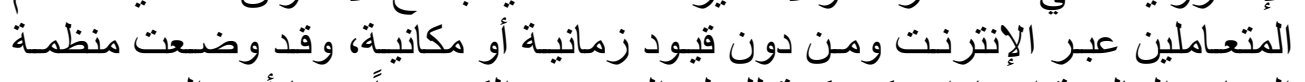

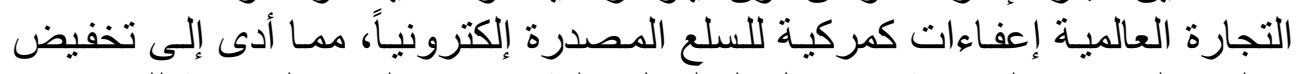

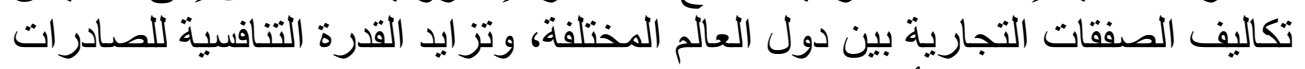

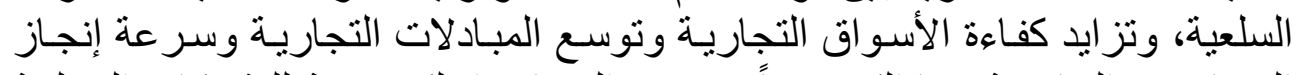

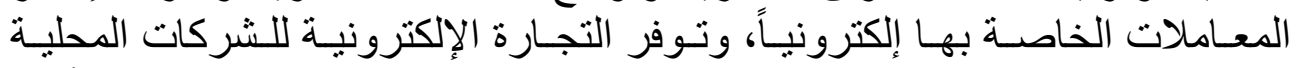

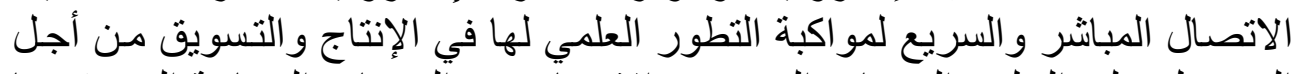

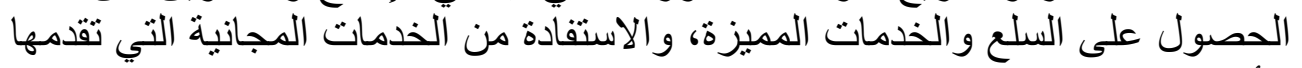

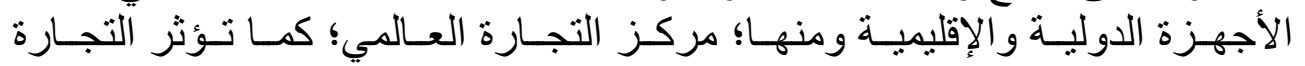

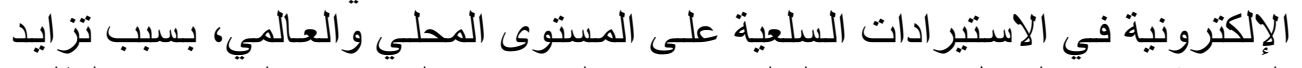

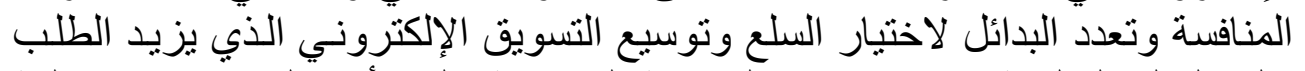

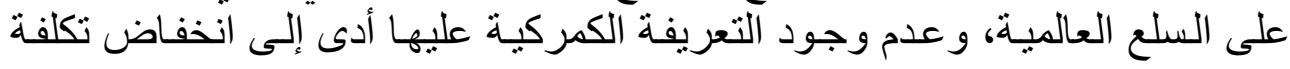




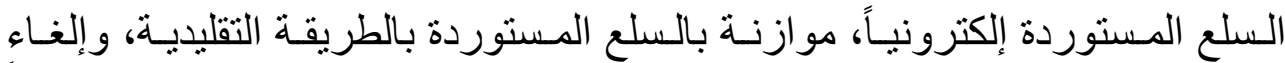

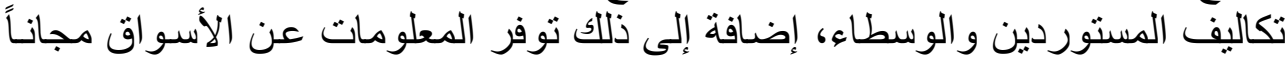

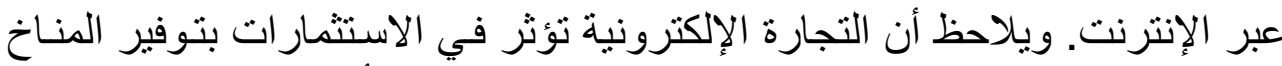

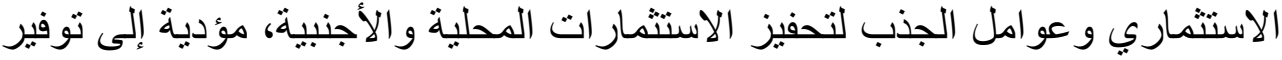

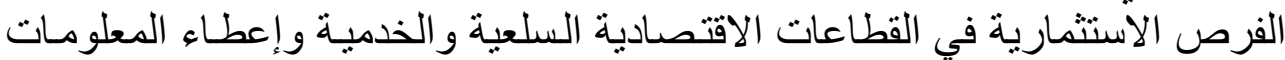

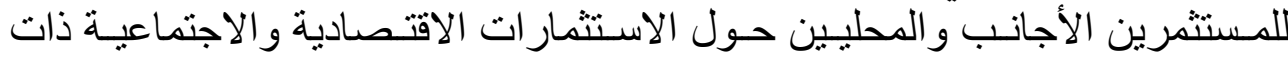

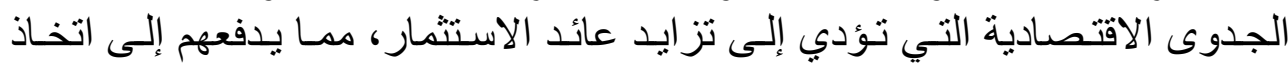

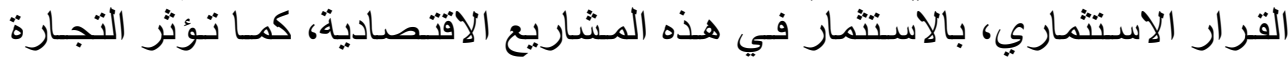

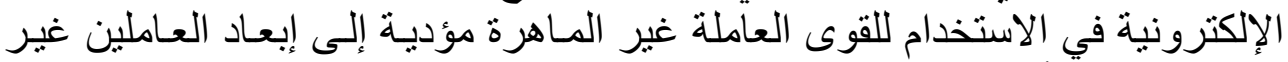

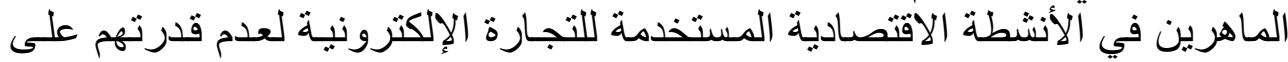

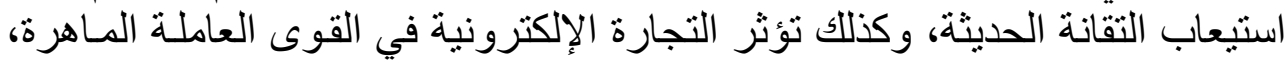

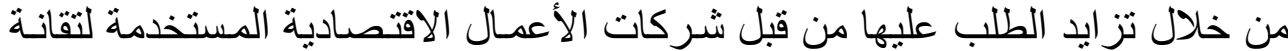

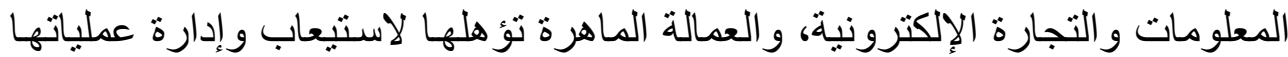

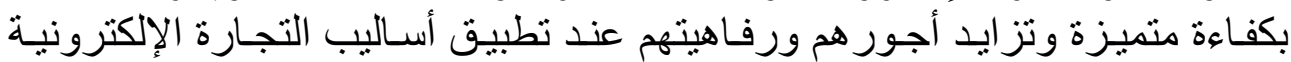

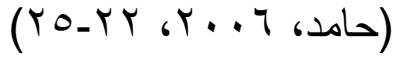

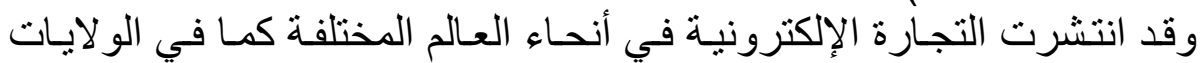

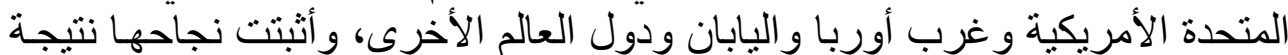

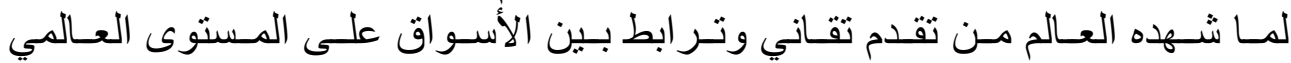
(www.lslam online)

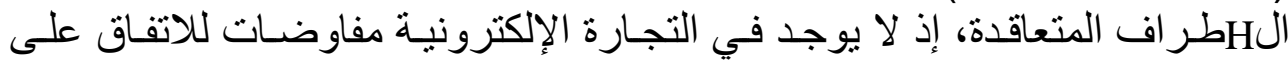

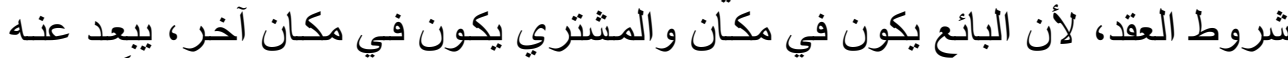

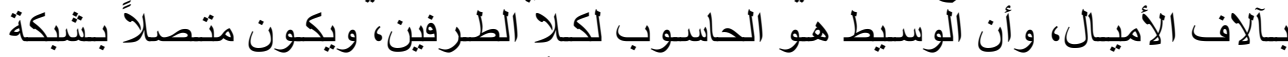

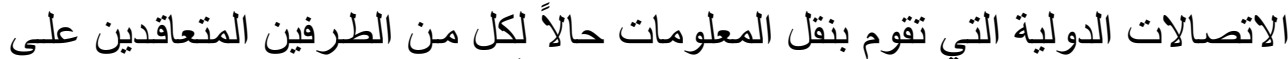

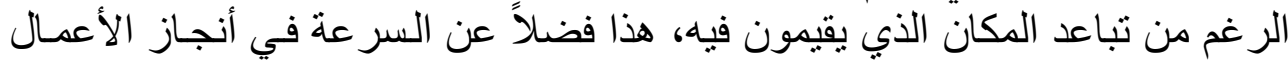

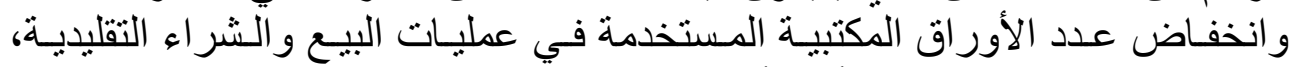

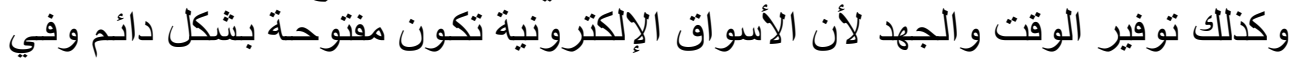

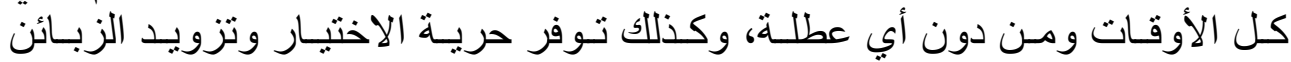

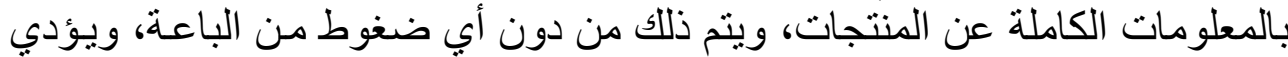

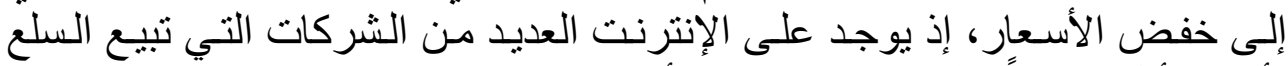

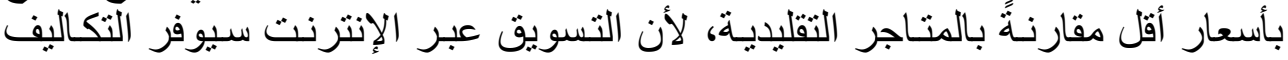

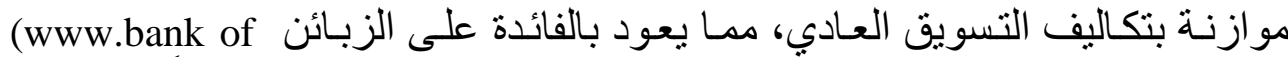

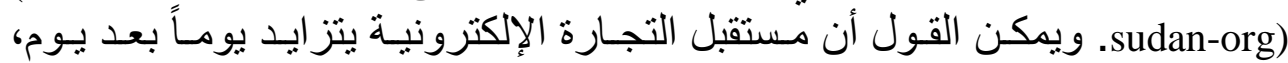

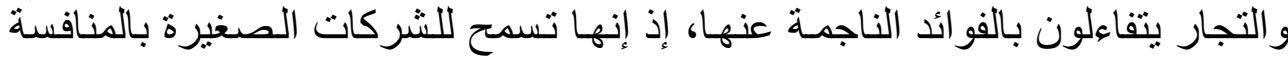

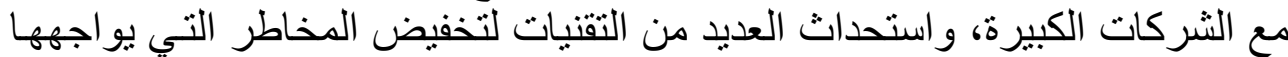

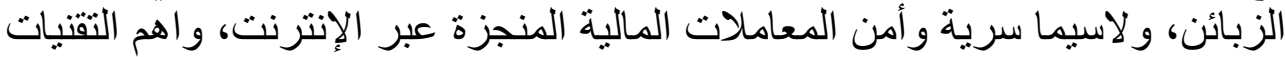

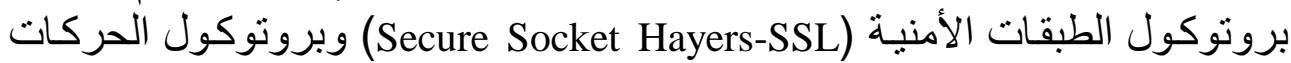

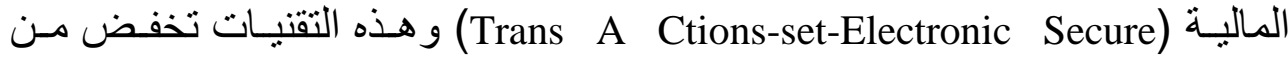




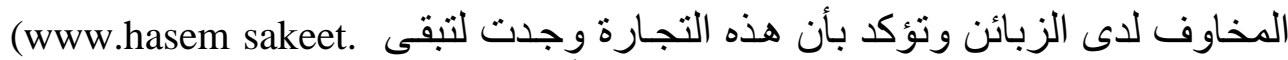
(8m. com)

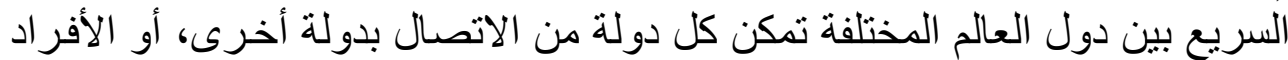

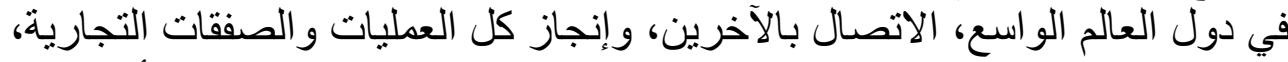

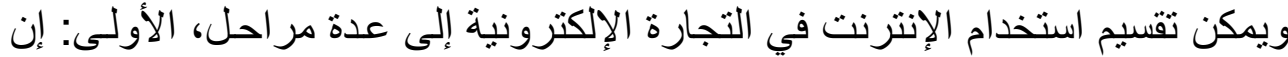

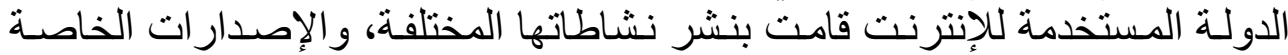

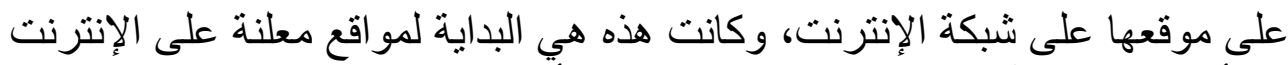

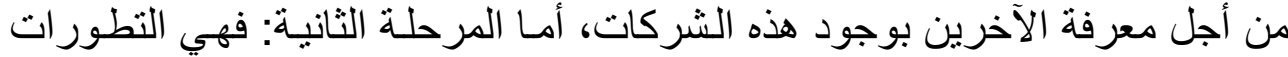

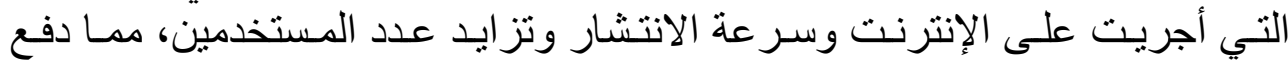

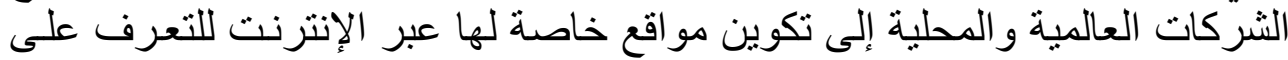

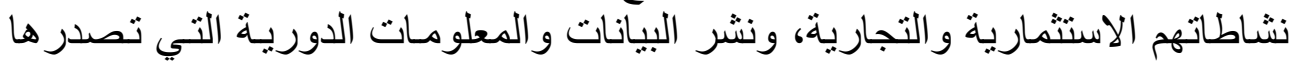

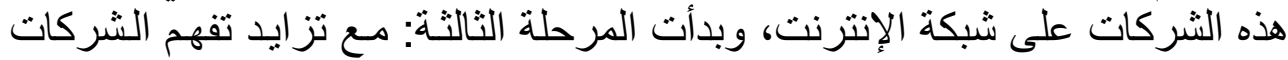

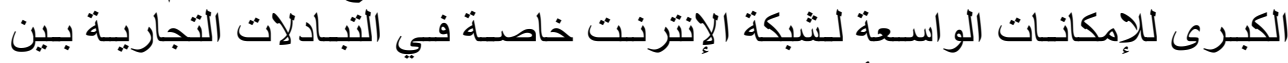

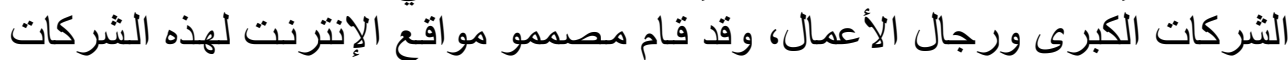

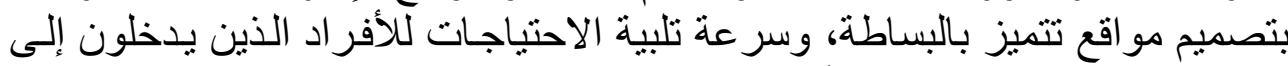

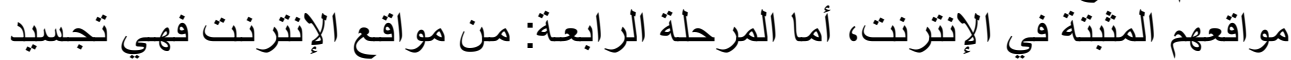

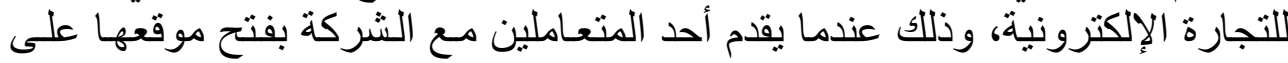

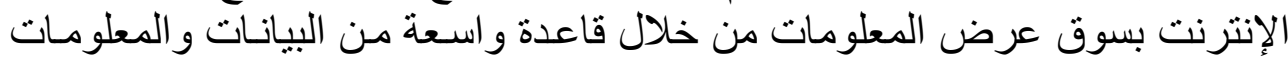

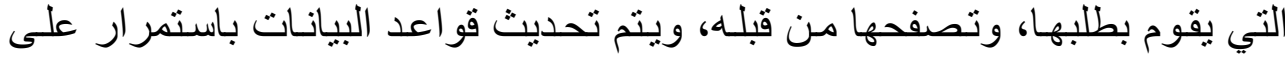

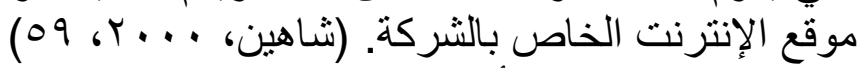

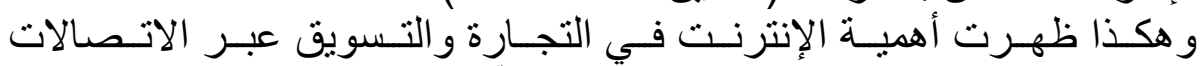

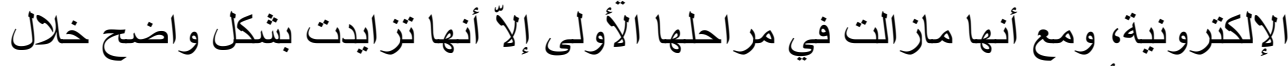

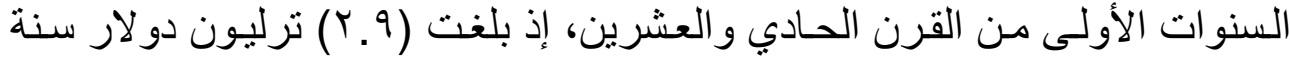

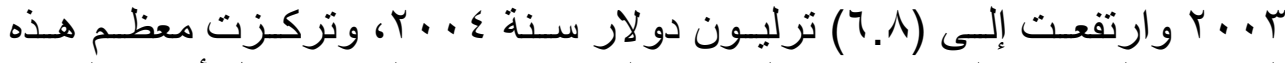

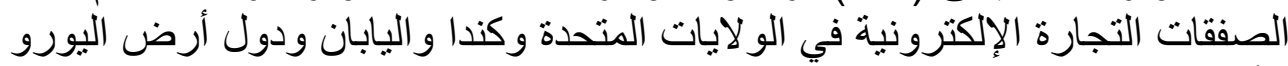

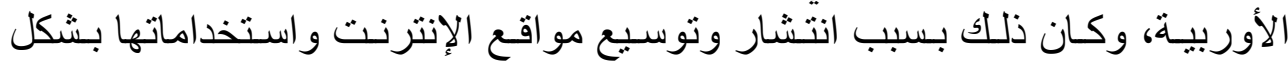

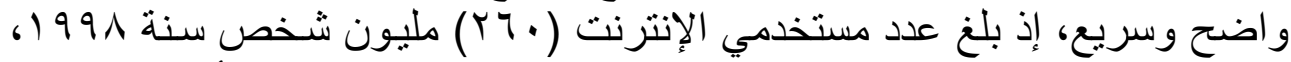

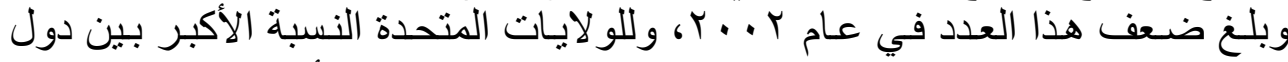

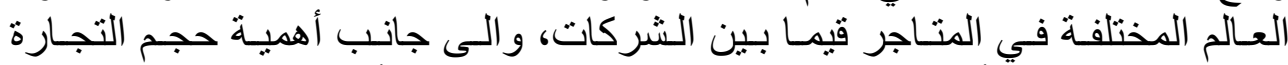

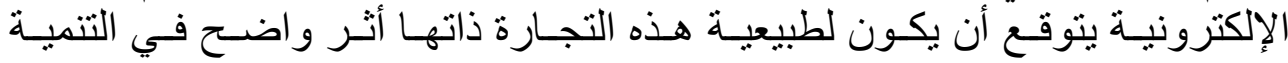

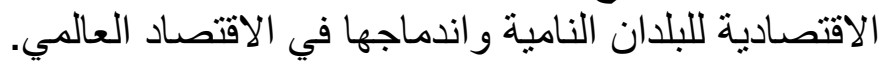

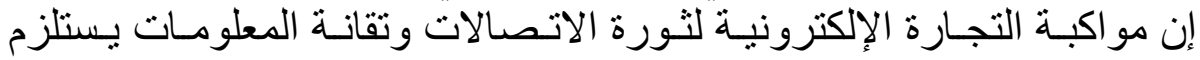

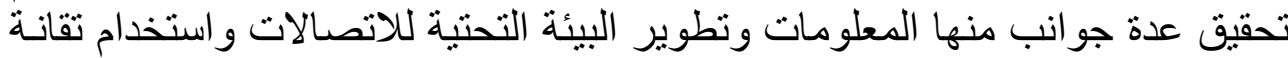

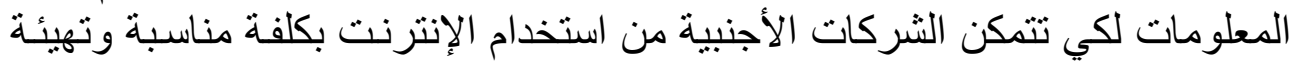

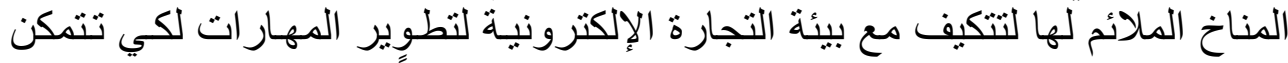
من استخدام تقانة المعلومات في التجارة الإلكترونية، فضلاً عن تقديم المساعدة للوفاء لتهاء 
بمتطلبات الدقاييس الدوليـة للسلع و الخدمات وتسهيل العمل التجاري في الأسواق

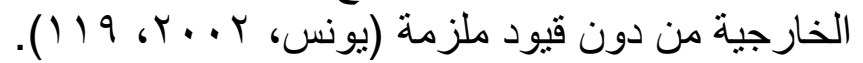

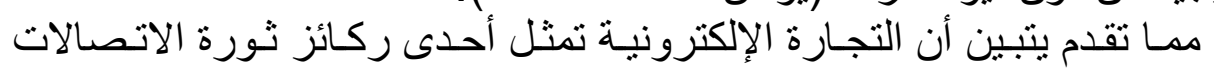

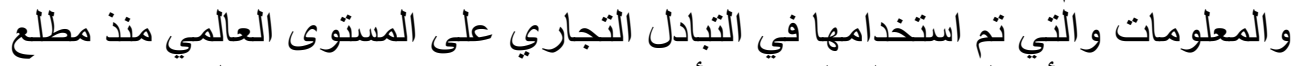

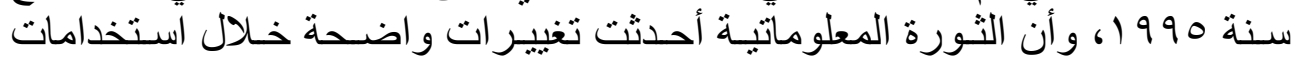

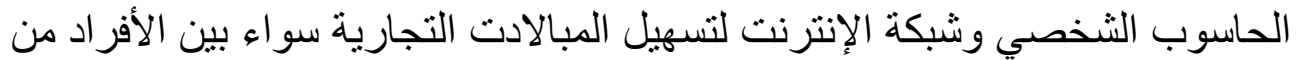

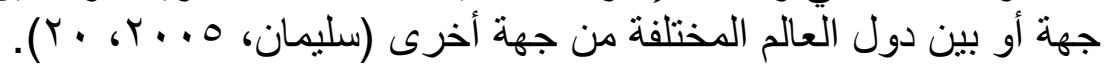

المحور الثاني- اتجاهـات تطور التجارة الإكترونيـة لدول العـالم المختلفة ومنهـا العربية

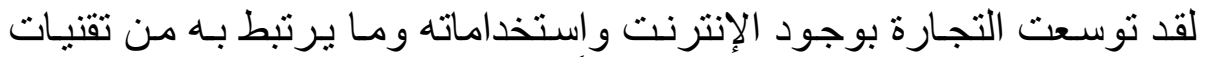

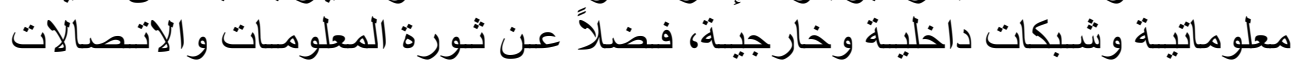

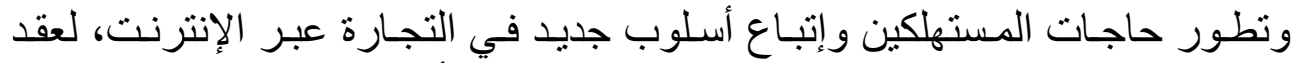

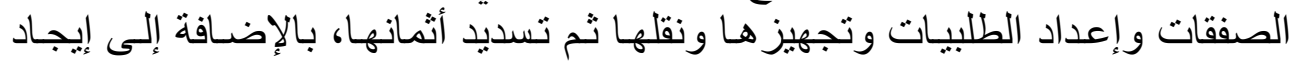

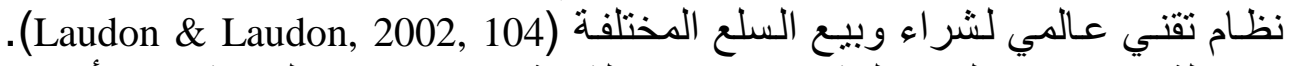

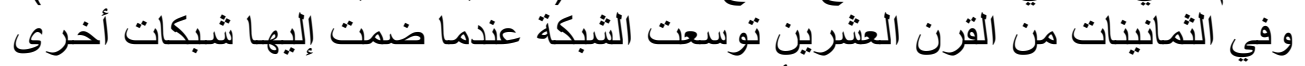

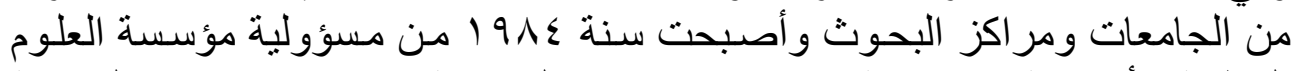

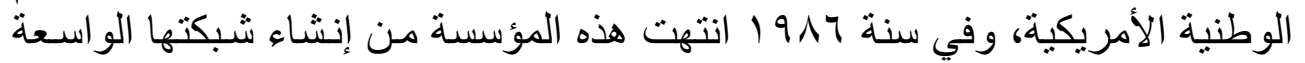

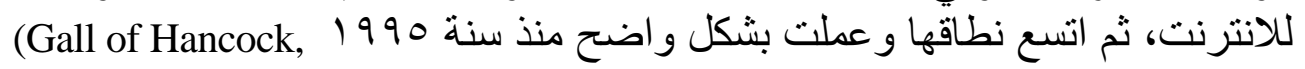
2002, 236)

وقد أكد الاقتصاديون على المز ايا الواسعة لشبكة الإنترنت، لأنها وسيلة فائقة

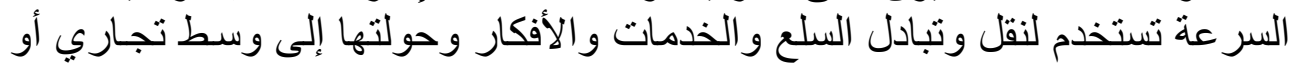

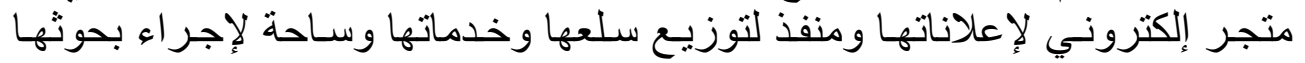

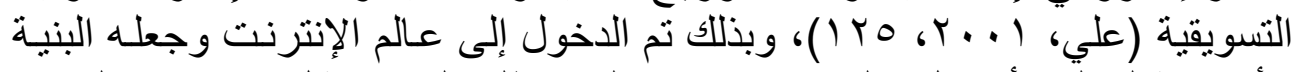

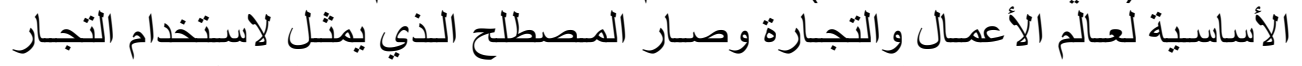

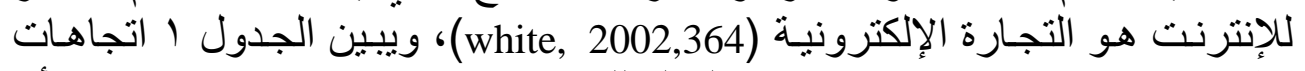

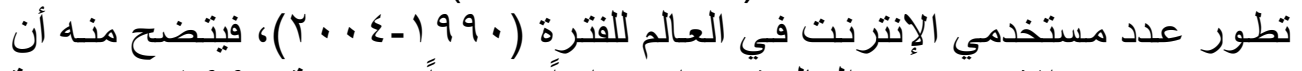

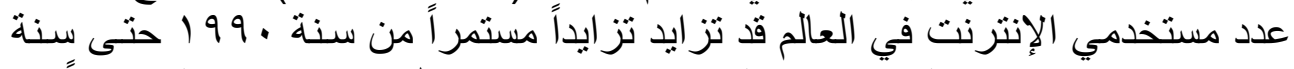

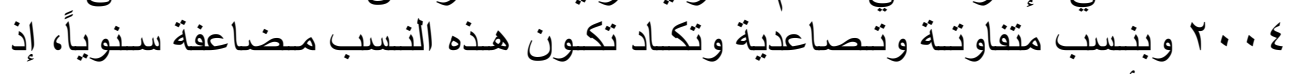

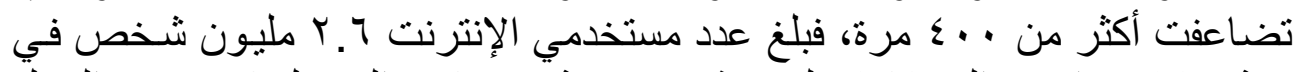

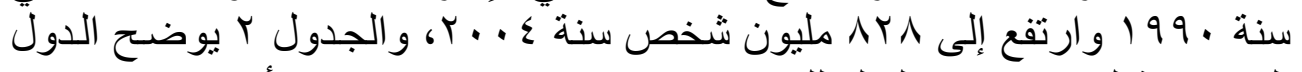

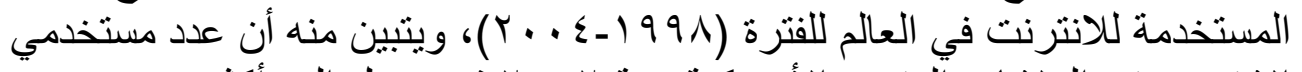

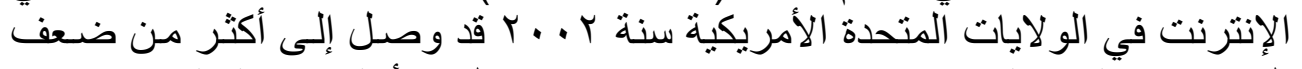

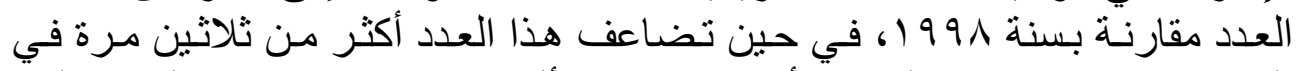

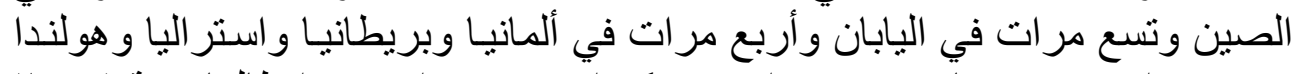

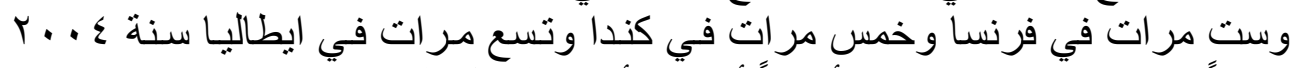

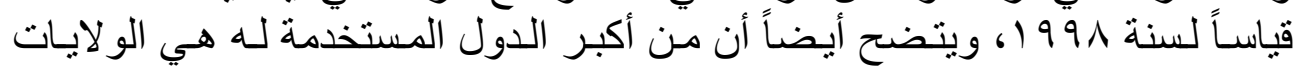


المتحدة الأمريكية ثم تليها الصين و اليابان و ألمانيا، وذللك بسبب التطور الحاصل في التجارة الإلكترونية.

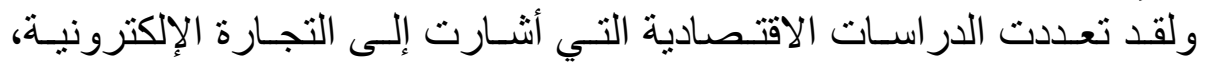

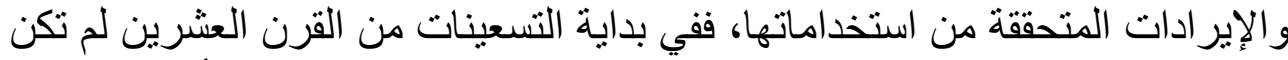

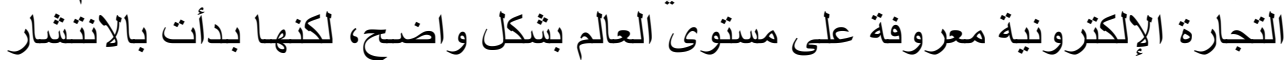

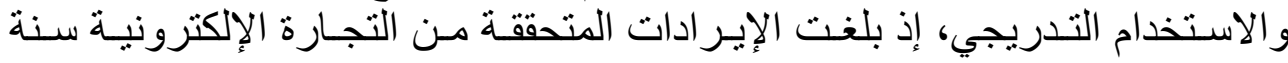

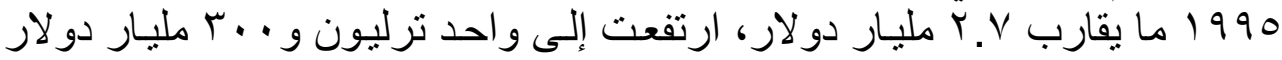

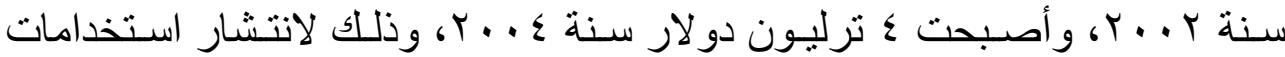

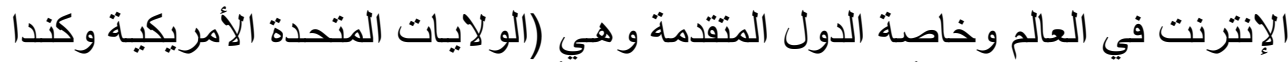

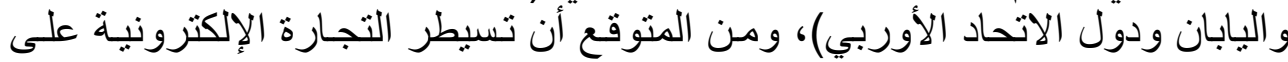

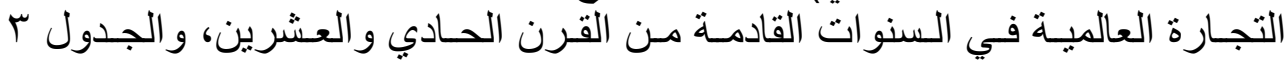

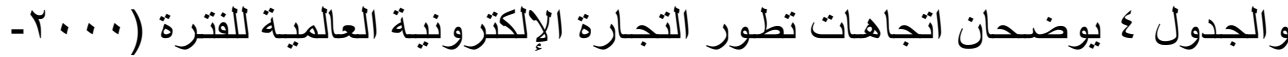

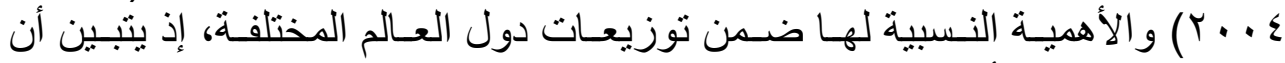

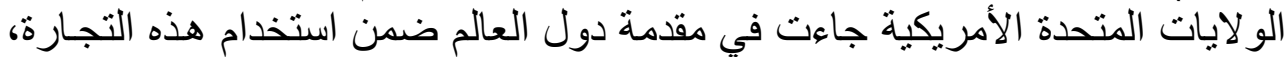

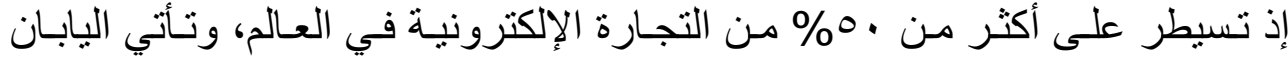

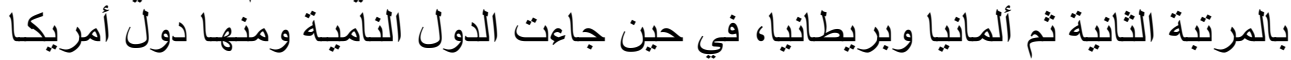
اللاتينية وبقية دول العالم في المرتبة الأخيرة، لأن تطبيقات التجارة الإلكترونية فيها

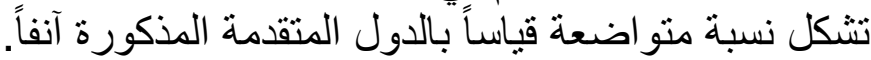

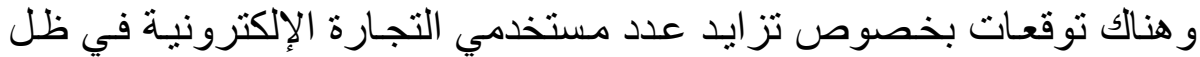

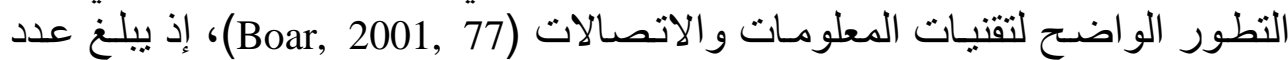

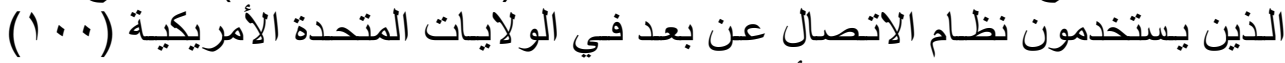

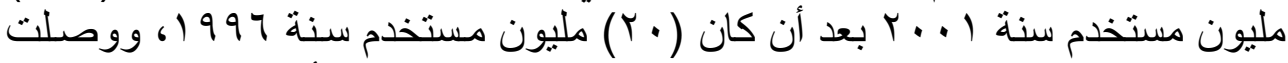

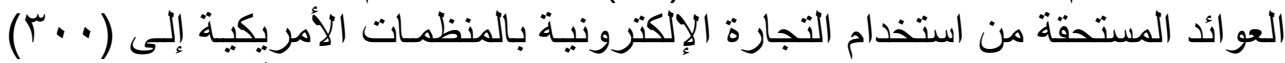

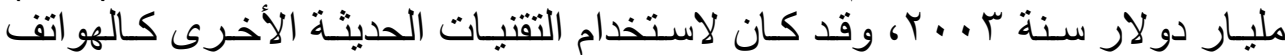

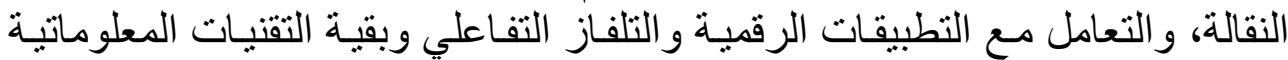

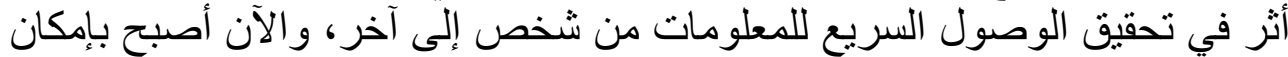

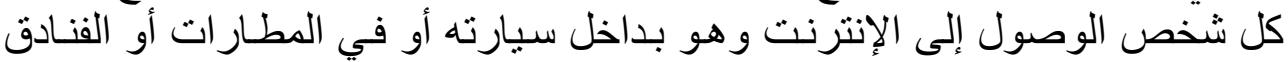

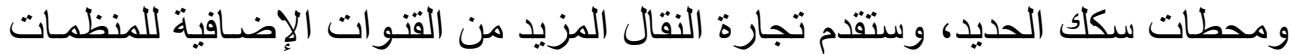

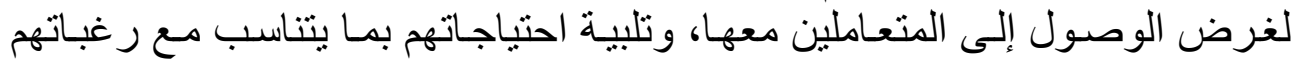

واحتياجاتهم المختلفة. (haudon \& haudon, 2002, 114)

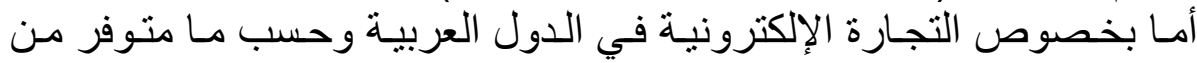

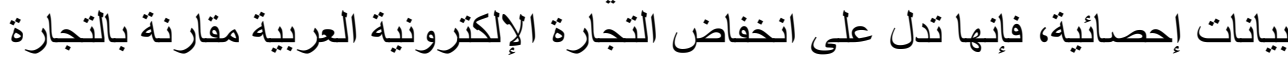

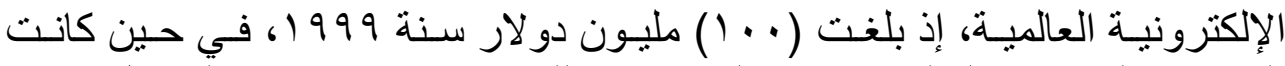

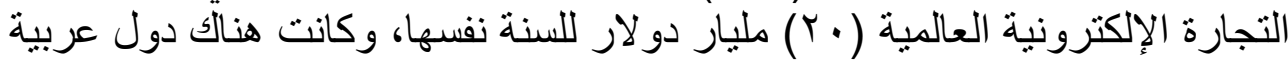

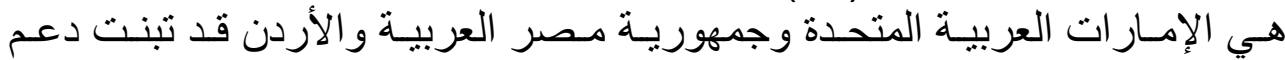

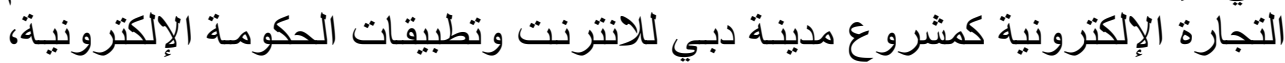

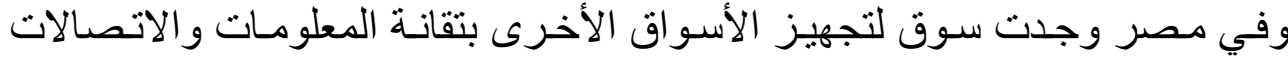


بصورة مباثرة من الأسواق عالمياً، ويمكن اعتبار مصر نقطة تحول نوعية مستقبلية

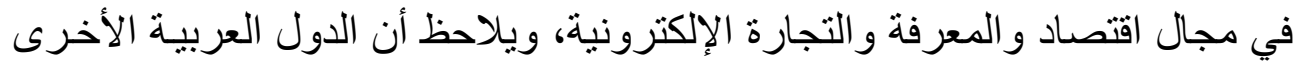

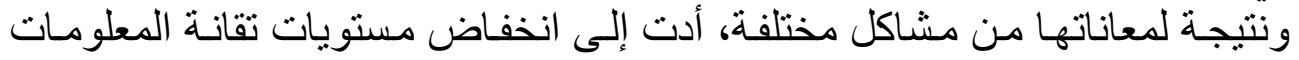

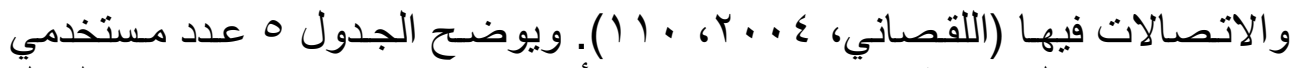

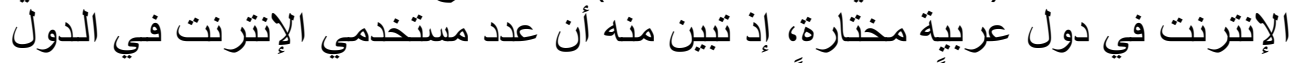

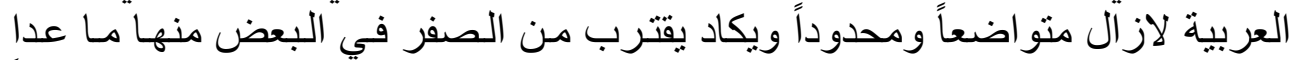

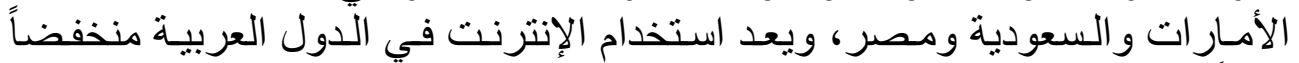

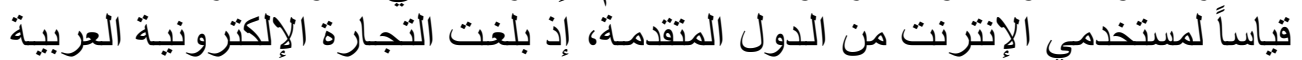

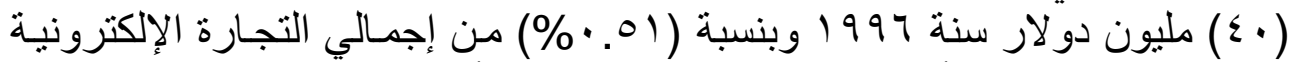

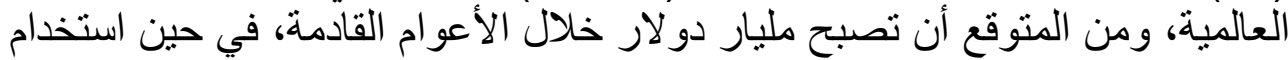

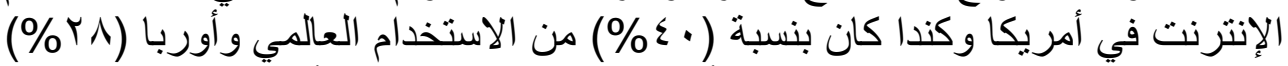

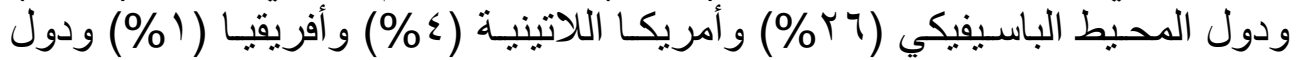

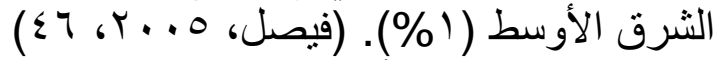

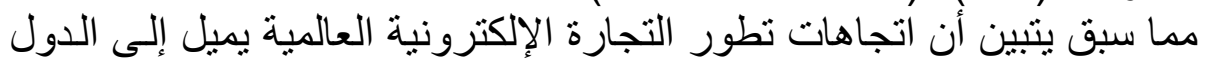

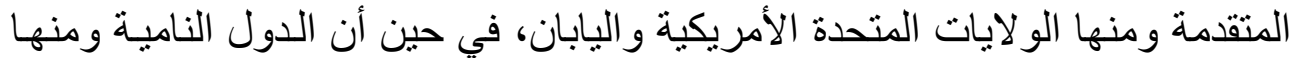
العربية تشخل نسبة متو اضعة من هذه التجارة

\section{الجدول المترة}

\begin{tabular}{|c|c|}
\hline عدد مستخلمي الإنترنت (مليون مستخدم) & السنة \\
\hline$r .7$ & 199. \\
\hline$\varepsilon . \varepsilon$ & 1991 \\
\hline 7.9 & 1994 \\
\hline 9.5 & 1994 \\
\hline 17 & 1995 \\
\hline$\Gamma \varepsilon$ & 1990 \\
\hline $0 \leqslant$ & 1997 \\
\hline 9. & $199 \mathrm{~V}$ \\
\hline
\end{tabular}




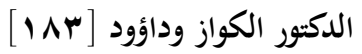

\begin{tabular}{|c|c|}
\hline $1 \leq 9$ & 1991 \\
\hline$r r$ & 1999 \\
\hline$r 11$ & $r \ldots$ \\
\hline$r q 1$ & $r \ldots r$ \\
\hline $0 \ldots$ & $r \ldots r$ \\
\hline$r 0$ & $r \ldots r$ \\
\hline$\Lambda r \Lambda$ & $r \ldots \varepsilon$ \\
\hline
\end{tabular}

Source: I - T - u, 2002 (www.itu-int)

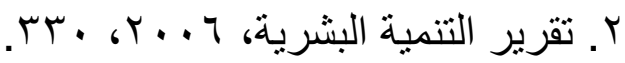

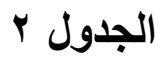

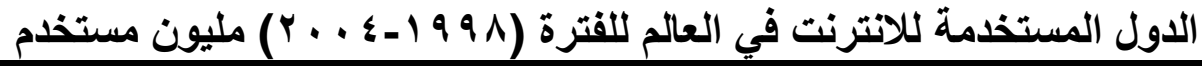

\begin{tabular}{|c|c|c|c|}
\hline$Y \ldots \varepsilon$ & $r \ldots r$ & 1991 & الدولة \\
\hline 117.0 & $10 \leqslant .7$ & $v \cdot .1$ & أمريكا \\
\hline $90 . \varepsilon$ & 9.5 & r.r & الصين \\
\hline Vo.- & rr.- & $\Lambda . \wedge$ & اليابان \\
\hline$\varepsilon 1 . r$ & r. & $1 \cdot . r$ & المانيا \\
\hline$r V . T$ & rr.r & 1.9 & بريطانيا \\
\hline rq & $1 T . Y$ & $r .1$ & ايطاليا \\
\hline ro.- & rז.r & ร.- & فرنسا \\
\hline$Y \cdot .-$ & 1 I.r & $\varepsilon .-$ & كندا \\
\hline Ir.A & 0.1 & T. $\varepsilon$ & استر اليا \\
\hline $9 . \varepsilon$ & $V .7$ & r.o & هو لندا \\
\hline
\end{tabular}

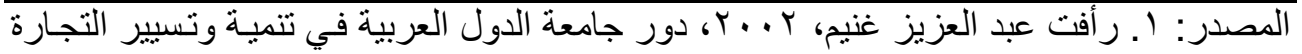

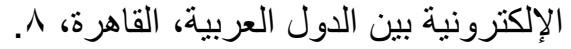

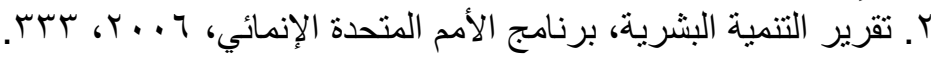

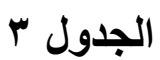

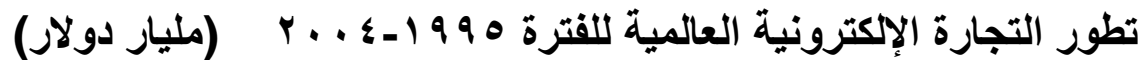

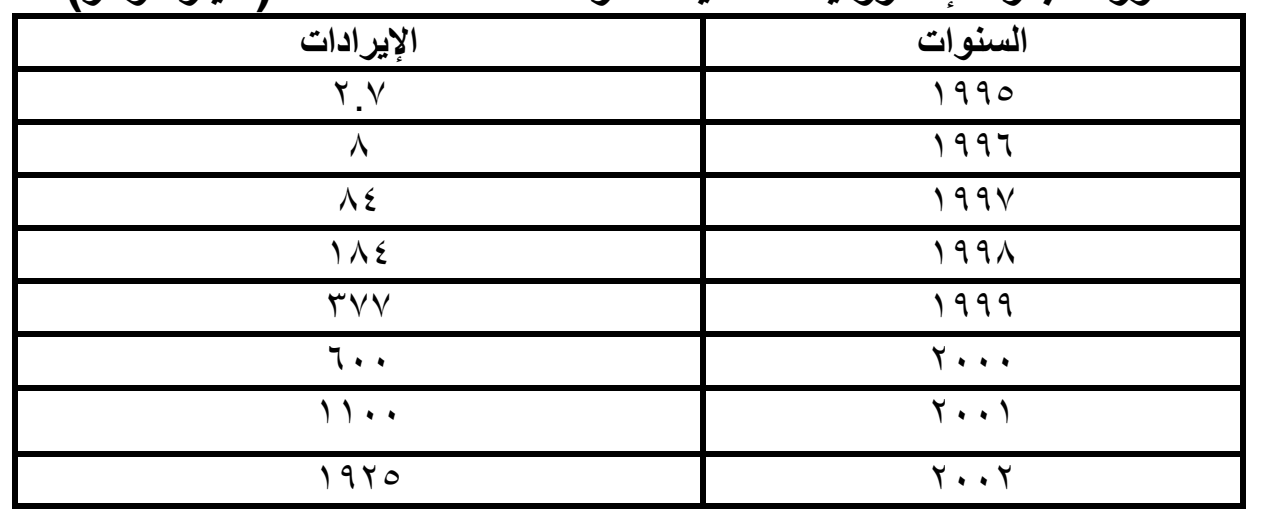




\begin{tabular}{|c|c|}
\hline rq0. & $r \ldots r$ \\
\hline $0 \leq V$. & $r \ldots \varepsilon$ \\
\hline
\end{tabular}

1. I-un cta d1 Electronic Commerce and Develop ment, 2001, 2002.

$$
\text { المصدر: }
$$

\section{PX.}

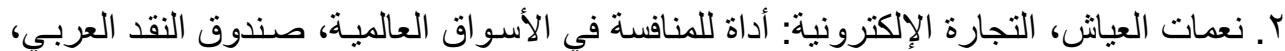

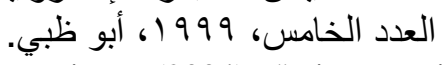

3. shellu, Gary, B, clash man, Thomas, J, \& vermat, mistye (2004), "Dic covering.

Computers - A G ate way to information web, "thom pson course techaolog Y, 10, 45.

\section{الجدول \\ تطور التجارة الإكترونية لاول العالم المختلفة والأهمية النسبية

\begin{tabular}{|c|c|c|c|c|c|c|c|c|c|c|}
\hline \multicolumn{5}{|c|}{ الأهمية النسبية \% } & \multirow{2}{*}{$r \ldots \varepsilon$} & \multirow{2}{*}{$r \ldots r$} & \multirow{2}{*}{$r \ldots r$} & \multirow{2}{*}{$r \ldots 1$} & \multirow{2}{*}{$r \ldots$} & \multirow{2}{*}{ الدولة } \\
\hline$r \cdots \varepsilon$ & $r \ldots r$ & $r \ldots r$ & $r \ldots 1$ & $r \ldots$ & & & & & & \\
\hline O^.V & 11.0 & VT.o & $V V .0$ & $\wedge 1 . \wedge$ & 119 & rAIV.r & $1 \leq 11 . r$ & $\wedge \leq 7.1$ & $\varepsilon \wedge \wedge . \vee$ & امريكا \\
\hline 17.1 & 9.1 & V.o & 7.0 & $0 . r$ & $\Lambda \wedge \cdot . r$ & & $1 \leq 7 . \wedge$ & $7 \varepsilon . \varepsilon$ & 1). 9 & اليابان \\
\hline V.I & $0 . r$ & $0 . Y$ & $\varepsilon . \varepsilon$ & r.o & т人т & 11.1 & $1 \cdot r$ & $\leqslant 7 . \leqslant$ & $r \cdot .7$ & المانبا \\
\hline r. 9 & Y.V & r.o & T.z & r. 9 & 17. & 1.9 .7 & 71 & rᄉ & $1 V . \varepsilon$ & كندا \\
\hline $0 . r$ & $\varepsilon . r$ & $\varepsilon . r$ & r.o & $r \cdot \Lambda$ & 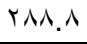 & 170.7 & NT.r & rᄉ.o & IV.r & بريطانيا \\
\hline r.V & Y. $\varepsilon$ & r.l & $1 . \varepsilon$ & $1 .$. & $r \cdot V .7$ & $97 . V$ & 19.9 & $1 \leq$ & 0.7 & استر اليا \\
\hline r.V & Y. .7 & r.o & r.r & 1.7 & $r \cdot 7 . \varepsilon$ & $1 \cdot \varepsilon . \wedge$ & $\sum 9.1$ & YY.I & 9.9 & فرنسا \\
\hline $1 . \varepsilon$ & $\because \wedge$ & $\because V$ & $\because 0$ & $\because 7$ & $\wedge 1 . \wedge$ & $M . \wedge$ & $1 T . V$ & $7 . r$ & r. & أمريكينية \\
\hline $1 . r$ & $1 . r$ & $\because v$ & .0 & .0 & $v \cdot .1$ & $\leq 9.7$ & 17 & $7 . r$ & $0 . V$ & بقية العالم \\
\hline $1 \cdots$ & $1 \cdots$ & $1 \cdots$ & $1 \ldots$ & $1 \cdots$ & $0 \leqslant V$. & r9o. & 1940 & $11 \ldots$ & 7. & المجموع \\
\hline
\end{tabular}

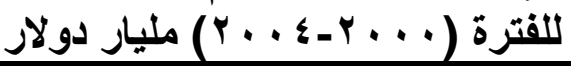

المصدر: فاروق احمد يونس، تطور التجارة الإلكترونية اليوم وغداً، مجلة دراسـات اقتصادية، العدد ؛ ؛

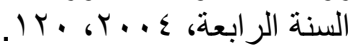

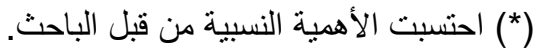

\section{الجدول ه}

\begin{tabular}{|c|c|c|c|}
\hline عدد مستخدمي الإنترنت & عدد مستخدمي الإنترنت لكل & عدالمليون السكان & اسم الدولة \\
\hline $7 \pi 0$ & $T \leqslant \varepsilon$ & $Y .7$ & الكويت \\
\hline $1 \leqslant 9$ & TाT & $\because V$ & البحرين \\
\hline IV. & TIT & $\cdot . \wedge$ & قطر \\
\hline $1 . \mu \wedge$. & Mr & $\varepsilon . r$ & الأمار ات \\
\hline$r \xi Y$ & $9 V$ & r.o & عمان \\
\hline$r .0$ & ry & $0 . V$ & ليبيا \\
\hline $1.0 \wedge \varepsilon$ & 77 & $r \varepsilon .$. & السعو دية \\
\hline $09 \varepsilon$ & 179 & r.o & لبنان \\
\hline
\end{tabular}

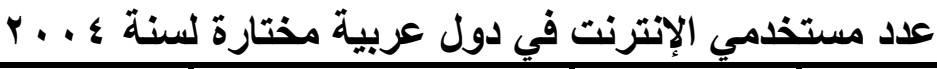




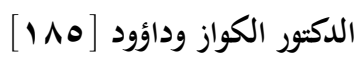

\begin{tabular}{|c|c|c|c|}
\hline 717 & 11. & 0.7 & الاردن \\
\hline$\wedge \varepsilon$. & $\Lambda \varepsilon$ & 1. & تونس \\
\hline$\Lambda \varepsilon Y$ & $r 7$ & T. & الجز ائر \\
\hline$r . q r$. & $0 \leqslant$ & VY. $T$ & مصر \\
\hline T.TYV & 118 & TI & المغرب \\
\hline 1.1147 & ru & ro.o & السودان \\
\hline 9 & IT &.$\wedge$ & جيبوتي \\
\hline $1 \wedge \pi$ & 9 & $r . r$ & اليمن \\
\hline Vo. & $r$. & ro & العراق \\
\hline
\end{tabular}

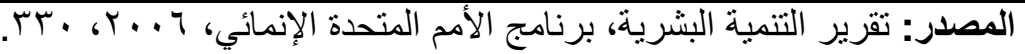

\section{الاستنتاجات والتوصيات \\ توصل البحث إلى مجمو عة من الاستنتاجات و التوصيات وهي كالآتي:

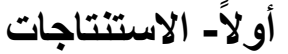

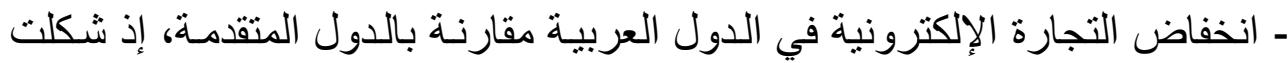

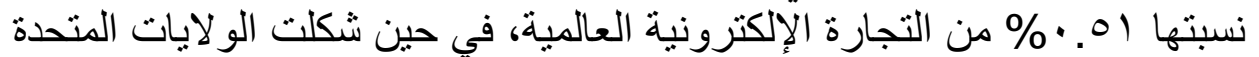

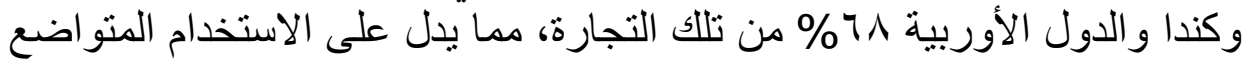

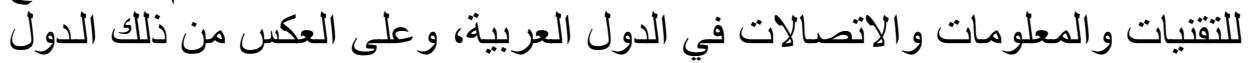

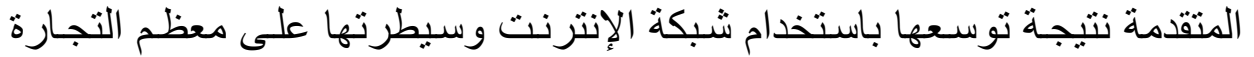
الإلكترونية. - اتضح أن استخدام التجارة الإلكترونية ووسائل الاتصال الحديثة يسهم في العملية

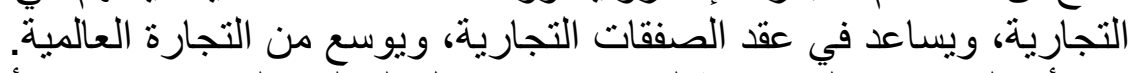

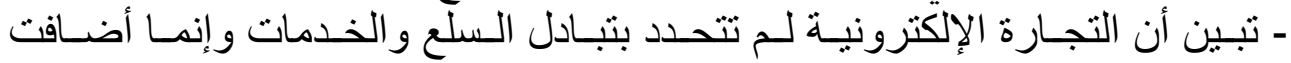

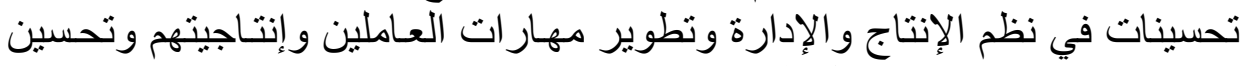
العلاقات التجارية بين الدول المتئقدمة. ثناتياً التوصيات

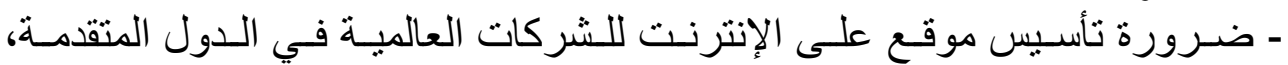

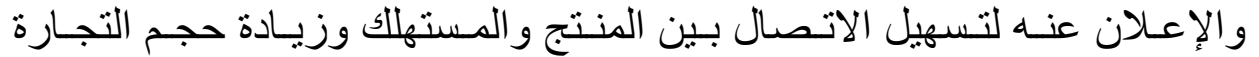

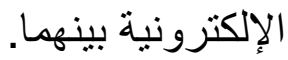

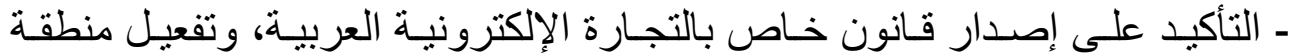

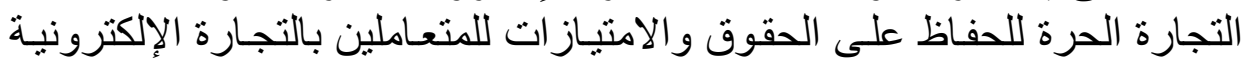

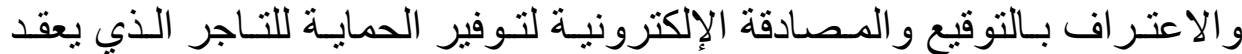

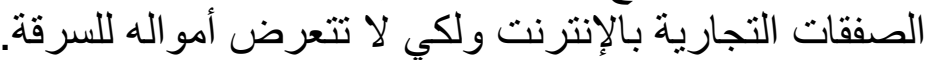

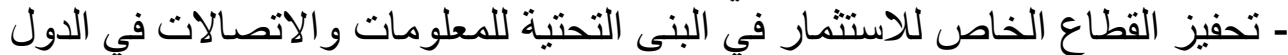

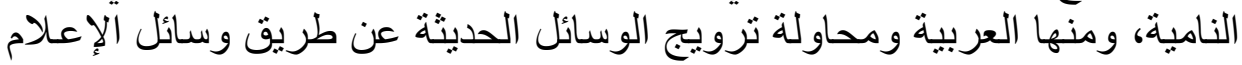

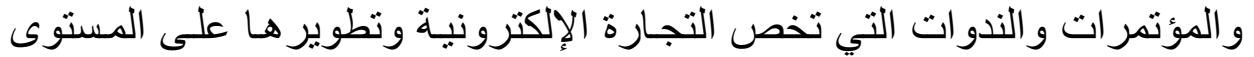

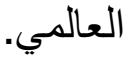




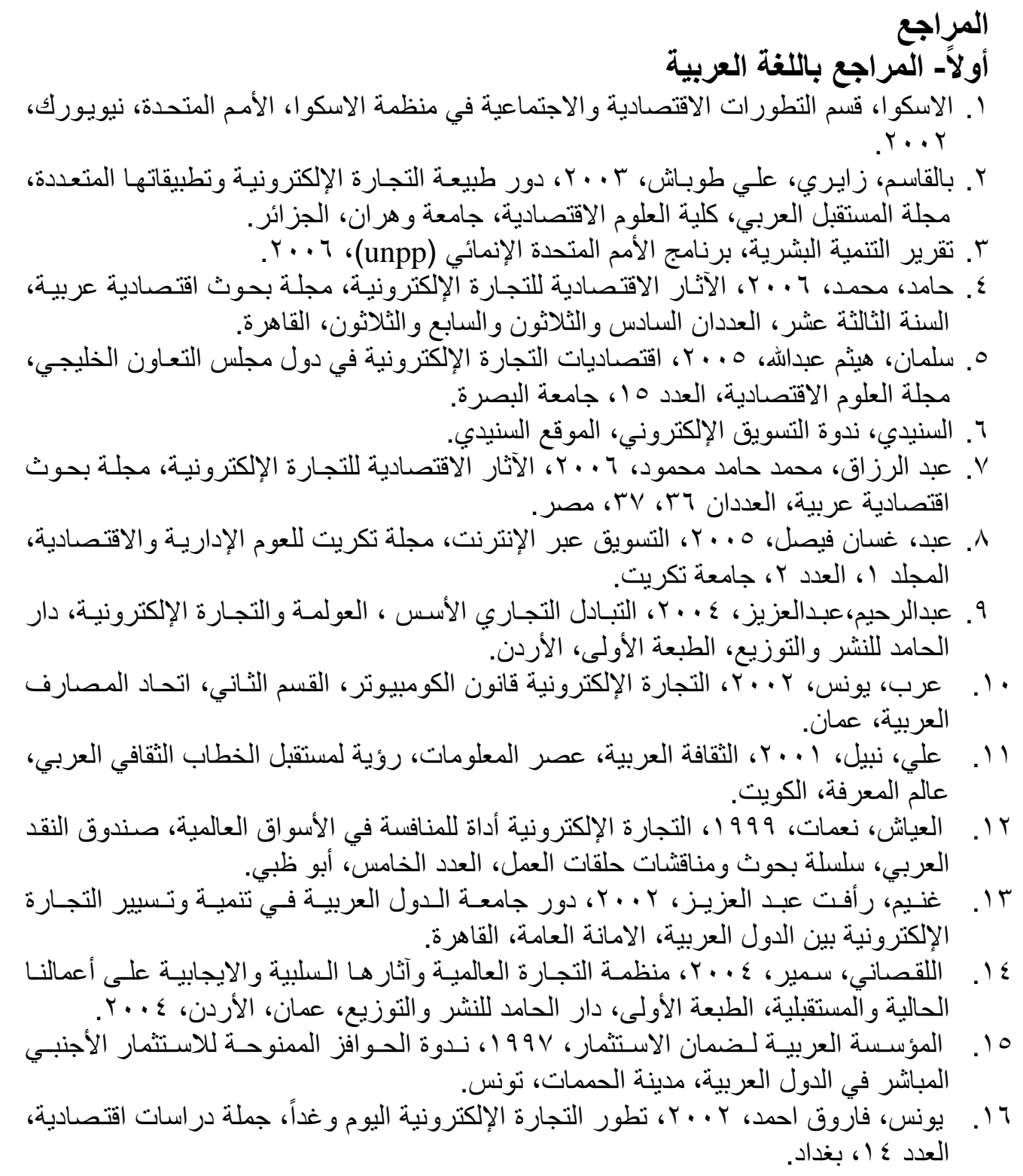

\section{ثانياً- المراجع باللغة الإجنبية}

1. Alter, steven, 1999, information System manag ement perspe ctwe, $3^{\text {rd }}$, ed- A ddison Wesley, hnc, Educcdion published new york.

2. Boar, Bernard, 2001, "The Art of strategic planning for in formation Technology", $2^{\text {nd }} e d$, united states of America.

3. Gallo, Michael A. \& Hancock, William m., 2002, computer communications \& Net working Technologies", Thomson Learnims, united states of America,

4. http//www. Islam - online Net / Arabic / . Ecommec / to pi co3 - shtml.

5. ITu, www. Ltu - int.

6. Laudon, Kenneth C. \& Laudon, Jane p., 2002, " manag ement information System 7th ed, prentice - "a" new Jersey. 


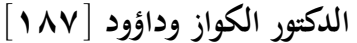

7. p: www. Book of sudan. Org / Arabic / period / masrafi / vol. 26 / masrafi 26 ht mhtt.

8. samiee, saeed, 1998, "the intern et \& internation al marketing: is there A fiy?" Jim, vol12 , no. 4 , Jhon wiley \& sons.

9. Shelly, Garry, B, clash man, Thomas, J, \& vermait, misty E., 2004, "Dic covering Computers - Ag ate way to information web, "the m son course Technology".

10. unctad, 2001-2002 "Electronic commerce and Develop ment Report", united N afions, New york and Geneva.

11. unctad, 2002, Hand Book of Statistics united Nations, New york and Geneva.

12. white, curt M., 2002, "Data communications \& computer Net Works, A Business users Approach" $2^{\text {nd }}$ E d., Thomson Course Technology, united states of American.

13. wto 2002, Annual year book. www. Wto-org.

14. www .britinlca.com / eb / pho? Search, e.

15. www. Al anhdi - ws.

16. www. Ecommece-gov. /p/ . Ecommec / de.

17. www. Eu. Org / ecommerce / 112 / pdf.

18. www. Hazemsake et $-8 \mathrm{~m}-\mathrm{com}$.

19. www. world Bank. Org public sector / tay / ecommerce-htm. 